\title{
A concurrent logical framework I: Judgments and properties
}

\author{
Kevin Watkins Iliano Cervesato Frank Pfenning \\ David Walker
}

March 2002, revised May 2003

CMU-CS-02-101

School of Computer Science

Carnegie Mellon University

Pittsburgh, PA 15213

This research was sponsored in part by the National Science Foundation under the title "Meta-Logical Frameworks," grants CCR-9619584 and CCR-9988281; and in part by NRL under grant N00173-00-C2086. Iliano Cervesato was partially supported by NRL under contract N00173-00-C-2086 and by NSF grant INT98-15731. David Walker was partially supported by the Office of Naval Research under the title "Efficient Logics for Network Security," grant N00014-01-1-0432; by DARPA under the title "Scaling ProofCarrying Code to Production Compilers and Security Policies," Contract No. F30603-99-1-0519; and by Microsoft Research. A part of this work was completed while David Walker was a post-doctoral fellow at Carnegie Mellon University. 


\begin{abstract}
The Concurrent Logical Framework, or CLF, is a new logical framework in which concurrent computations can be represented as monadic objects, for which there is an intrinsic notion of concurrency. It is designed as a conservative extension of the linear logical framework LLF with the synchronous connectives $\otimes, 1$, !, and $\exists$ of intuitionistic linear logic, encapsulated in a monad. LLF is itself a conservative extension of LF with the asynchronous connectives $\multimap, \&$ and $T$.

The present report, the first of two technical reports describing CLF, presents the framework itself and its meta-theory. A novel, algorithmic formulation of the underlying type theory concentrating on canonical forms leads to a simple notion of definitional equality for concurrent computations in which the order of independent steps cannot be distinguished. The new formulation of the framework constitutes an original contribution even for the LF fragment.

For many additional examples illustrating the use of the framework to specify and reason about object systems of interest, the reader is referred to the companion technical report on applications [CPWW02].
\end{abstract}

Keywords: logical frameworks, type theory, linear logic, concurrency 


\section{Contents}

1 Introduction $\quad 3$

2 A type theory with concurrent terms 4

2.1 A language of concurrent objects . . . . . . . . . . . . . 4

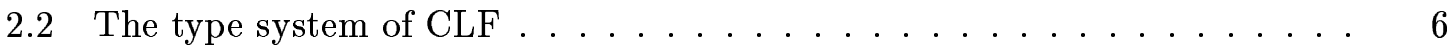

2.3 Related work . . . . . . . . . . . . . . . . 10

3 Representing concurrent systems $\quad 11$

3.1 An example: Petri nets with labeled tokens . . . . . . . . . . . . . . 11

3.2 Representing LPNs in CLF . . . . . . . . . . . . . . . . 13

3.3 Related work ........................ 15

4 Some meta-theoretic results $\quad 16$

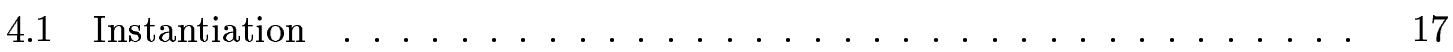

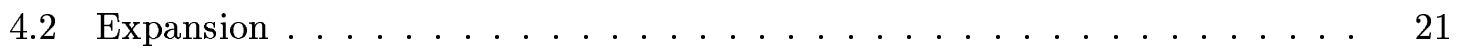

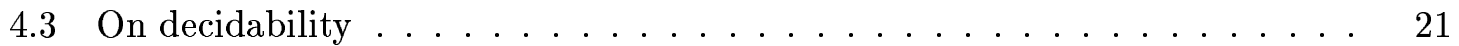

4.4 Composition . . . . . . . . . . . . . . . . . 23

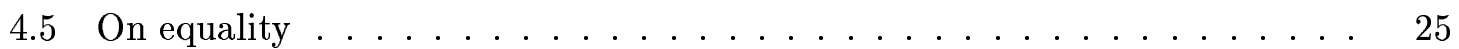

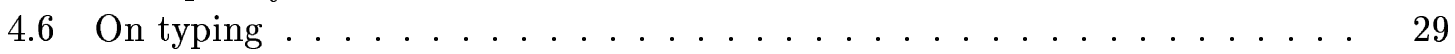

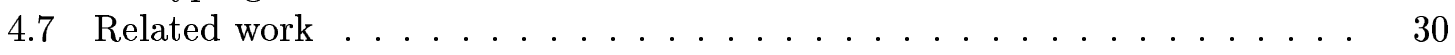

5 Conclusion $\quad 31$

A Syntax and judgments of CLF 32

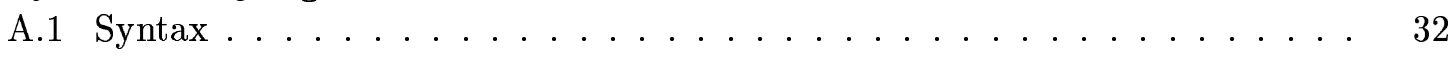

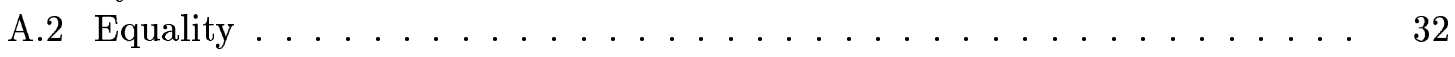

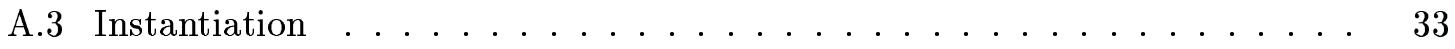

A.4 Expansion . . . . . . . . . . . . . . . . . . . . 34

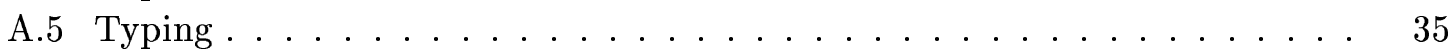





\section{Introduction}

A logical framework [Pfe01b, BM01] is a meta-language for the specification and implementation of deductive systems, which are used pervasively in logic and the theory of programming languages. A logical framework should be as simple and uniform as possible, yet provide intrinsic means for representing common concepts and operations in its application domain.

The particular lineage of logical frameworks we are concerned with in this paper started with the Automath languages [dB80] which originated the use of dependent types. It was followed by LF [HHP93], crystallizing the judgments-as-types principle. LF is based on a minimal type theory $\lambda^{\Pi}$ with only the dependent function type constructor $\Pi$. It nonetheless directly supports concise and elegant expression of variable renaming and capture-avoiding substitution at the level of syntax, and parametric and hypothetical judgments in deductions. Moreover, proofs are reified as objects which allows properties of or relations between proofs to be expressed within the framework [Pfe91].

Representations of systems involving state remained cumbersome until the design of the linear logical framework LLF [CP98] and its close relative RLF [IP98]. For example, LLF allows an elegant representation of Mini-ML with mutable references that reifies imperative computations as objects. LLF is a conservative extension of LF with the linear function type $A \multimap B$, the additive product type $A \& B$, and the additive unit type $\top$. This type theory corresponds to the largest freely generated fragment of intuitionistic linear logic [HM94, Bar96] whose proofs admit long normal forms without any commuting conversions. This allows a relatively simple type-directed equality-checking algorithm which is critical in the proof of decidability of type-checking for the framework [CP98, VC00].

While LLF solved many problems associated with the representation of stateful computations, the encoding of concurrent computations remained unsatisfactory. In this report, we demonstrate that the limitations of LLF can be overcome by extending the framework with a monad that incorporates the synchronous connectives $\otimes, 1$, !, and $\exists$ of intuitionistic linear logic. We call this new framework Concurrent LF (CLF).

The purpose of this report is to describe the language and meta-theory of CLF. Readers interested in examples of CLF representations can consult the companion report [CPWW02], which demonstrates the expressive power of CLF through a series of examples and, in particular, focuses on CLF's effectiveness for encoding concurrent programming paradigms.

Summary. The remainder of the report is organized as follows. Section 2 introduces the CLF type theory, including its syntax, equality judgments and typing judgments. Section 3 discusses how concurrent systems can be represented in CLF and how such representations improve on what is possible in LLF, and relates CLF to various other similar proposals. Section 4 describes CLF's instantiation and expansion operators, which permit the formulation of the framework without a notion of $\beta \eta$-conversion, and describes the key meta-theorems on equality and typing. It also compares the formulation of the type theory seen here with previous accounts of LF and related type theories. Finally, Section 5 offers some concluding remarks. 


\section{$2 \quad$ A type theory with concurrent terms}

\subsection{A language of concurrent objects}

In the LF tradition, the terms classified by types are called objects, and the terms classified by kinds are called type constructors, among which are the types. CLF has two categories of types: the asynchronous types $A$ and the synchronous types $S$. The asynchronous types include all the type constructors of LF and LLF, as well as a new monadic type constructor written $\{S\}$. The synchronous types, which are only allowed within the monad constructor, include further type constructors of intuitionistic linear logic, all of which have let-style elimination rules. Intuitively, the monad restricts the availability of these elimination forms so that the question of commutative conversions between the eliminations and other terms of the type theory does not arise. Sections 2.3 and 3.3 describe this in further detail. The "(a)synchronous" terminology is due to Andreoli [And92].

\section{DEFINITION 1 (TYPE CONSTRUCTORS)}

$$
\begin{aligned}
A, B, C:: & =A \multimap B|\Pi x: A . B| A \& B|\top|\{S\} \mid P \\
P & ::=a \mid P N \\
S:: & =S_{1} \otimes S_{2}|1| \exists x: A . S \mid A
\end{aligned}
$$

Asynchronous types

Atomic type constructors

Synchronous types

The asynchronous types include the dependent function type $\Pi x: A$. $B$, the linear function type $A \multimap B$, the additive product type $A \& B$, and the additive unit T. The monadic type $\{S\}$ acts as a coercion from the synchronous types into the asynchronous types. The atomic type constructors $P$ include type constructor constants $a$ and the type-level dependent application $P N$ (where $N$ is an object).

The synchronous types include the other type constructors new to CLF: the multiplicative product type $S_{1} \otimes S_{2}$, the multiplicative unit 1, and the dependent pair type $\exists x: A$. $S$. There is a trivial coercion from the asynchronous types into the synchronous ones. In addition, the exponential type ! of intuitionistic linear logic can be defined as a trivial dependent pair: $! A \equiv \exists x: A .1$.

The kind language of CLF is identical to that of LF and LLF. The symbol kind is also used on occasion to classify the valid kinds.

DEFINITION 2 (KINDS)

$$
K, L::=\text { type } \mid \Pi x: A . K \quad K i n d s
$$

We often write $A \rightarrow B$ instead of $\Pi x: A$. $B$ and $A \rightarrow K$ instead of $\Pi x: A$. $K$ when $B$ or $K$ respectively contains no free occurrence of $x$.

The CLF type theory inherits all the type and kind constructors of LF and LLF, and the corresponding objects. It differs, however, in that the syntax of CLF admits only those terms of LF and LLF that are $\beta$-normal and $\eta$-long - the canonical terms. This simplifies the meta-theory of CLF, and highlights the importance of the principle that LF representations always establish a compositional bijection between terms of an object language and the canonical objects of LF of a given type. Thus, in CLF, there is no notion of $\beta$ - or $\eta$-conversion, and every well-formed term can be regarded as "canonical." 


\section{DEFINITION 3 (OBJECts)}

$$
\begin{array}{rlrl}
N:: & =\hat{\lambda} x . N|\lambda x . N|\left\langle N_{1}, N_{2}\right\rangle|\langle\rangle|\{E\} \mid R & & \text { Normal objects } \\
R::=c|x| R^{\wedge} N|R N| \pi_{1} R \mid \pi_{2} R & & \text { Atomic objects } \\
E & :=\text { let }\{p\}=R \text { in } E \mid M & & \text { Expressions } \\
M:: & =M_{1} \otimes M_{2}|1|[N, M] \mid N & & \text { Monadic objects } \\
p::=p_{1} \otimes p_{2}|1|[x, p] \mid x & & \text { Patterns }
\end{array}
$$

The first two categories of object are the normal objects $N$ and the atomic objects $R$. These correspond to the quasi-canonical and quasi-atomic forms of LF object, respectively, as described by Harper and Pfenning [HP00]. A normal object is a series of constructors applied to atomic objects, while an atomic object is a series of natural-deduction style destructors applied to a variable $x$ or constant $c$. They include all the constructors and destructors of LF and LLF: the unrestricted function constructor $\lambda x . N$ and destructor $R N$; the linear function constructor $\hat{\lambda} x . N$ and destructor $R^{\wedge} N$; the additive pair constructor $\left\langle N_{1}, N_{2}\right\rangle$ and destructors $\pi_{1} R$ and $\pi_{2} R$; and the additive unit constructor \langle\rangle .

In addition, there is a constructor $\{E\}$ associated with the monadic type. The remaining categories of object, the expressions $E$ and monadic objects $M$, are associated with the monadic type and the additional linear type constructors $\otimes, 1$ and $\exists$. They include the monadic binding form let $\{p\}=R$ in $E$; the multiplicative pair constructor $M_{1} \otimes M_{2}$ and pattern $p_{1} \otimes p_{2}$; the multiplicative unit constructor 1 and pattern 1 ; and the dependent pair constructor $[N, M]$ and pattern $[x, p]$. The monadic binding form let $\{p\}=R$ in $E$ binds all the variables occurring in the pattern $p$ within the expression $E$. Hence, it subsumes the destructors for $1, \otimes$, and $\exists$. The purpose of the monadic type is to isolate these binding forms, which would otherwise have a catastrophic effect on the LF and LLF fragments of CLF, as explained in Section 3.2.

Terms which differ only in the names of their bound variables are considered to be the same. For the LF and LLF fragments of CLF, this is the only notion of equality: two terms are equal if and only if they are $\alpha$-equivalent. But expressions differ from the other categories of object in that they are subject to permutative conversions by which the monadic bindings can be reordered:

$$
\text { (let } \left.\left\{p_{1}\right\}=R_{1} \text { in let }\left\{p_{2}\right\}=R_{2} \text { in } E\right)=\left(\text { let }\left\{p_{2}\right\}=R_{2} \text { in let }\left\{p_{1}\right\}=R_{1} \text { in } E\right)
$$

Of course, this rule is subject to the proviso that the bindings be independent: $p_{1}$ and $p_{2}$ must bind disjoint sets of variables, no variable bound by $p_{1}$ can appear free in $R_{2}$, and vice versa. The reordering of monadic bindings is the mechanism by which CLF admits an intrinsic description of concurrent computations. We think of each let binding as a single computation step. Computation steps appearing in a single expression that are independent in the above sense can be thought of as occurring concurrently.

The notion of equality on CLF objects could be characterized as the least congruence relation including the above equation schema. The reason is that having separate syntactic classes of objects and expressions eliminates any need for commuting conversions. (We do not think of the permutative conversions as being commuting conversions.) But we prefer to define the framework's equality in a slightly different way. The definition relies on the subsidiary concept of a concurrent context. 


\section{DEFINITION 4 (CONCURRENT CONTEXTS)}

$$
\epsilon::=\_\mid \text {let }\{p\}=R \text { in } \epsilon \quad \text { Concurrent contexts }
$$

As usual, the notation $\epsilon[E]$ stands for the expression constructed by replacing the hole - in $\epsilon$ with $E$. Now equality can be defined as follows.

DEFINITION 5 (EQUALITY)

$E_{1}={ }_{\mathrm{c}} E_{2}$

[Concurrent equality]

$$
\frac{M_{1}=M_{2}}{M_{1}={ }_{\mathrm{c}} M_{2}} \quad \frac{R_{1}=R_{2} \quad E_{1}={ }_{\mathrm{c}} \epsilon\left[E_{2}\right]}{\left(\text { let }\{p\}=R_{1} \text { in } E_{1}\right)={ }_{\mathrm{c}} \epsilon\left[\text { let }\{p\}=R_{2} \text { in } E_{2}\right]} *
$$

$E_{1}=E_{2}$

[Expression equality]

$$
\frac{E_{1}={ }_{\mathrm{c}} E_{2}}{E_{1}=E_{2}}
$$

$N_{1}=N_{2} \quad R_{1}=R_{2} \quad M_{1}=M_{2} \quad P_{1}=P_{2}$

[Other equalities]

\section{(All congruences.)}

The judgment $E_{1}={ }_{\mathrm{c}} E_{2}$ holds when $E_{1}$ and $E_{2}$ represent the same underlying concurrent computation even though their syntactic representations may differ. The rule marked $\left(^{*}\right)$ is subject to the side condition that no variable bound by $p$ be free in the conclusion or bound by the context $\epsilon$, and that no variable free in $R_{2}$ be bound by the context $\epsilon$. (It is always possible to globally $\alpha$-convert terms being compared for equality in order to avoid running afoul of the side condition.)

Then the equality on expressions $E_{1}=E_{2}$ is simply defined to be concurrent equality. While the definition of concurrent equality is not presented symmetrically, it turns out to be symmetric. The judgments $N_{1}=N_{2}, R_{1}=R_{2}, M_{1}=M_{2}$, and $P_{1}=P_{2}$ are characterized by simple congruence rules for each syntactic form, not shown. In Section 4.5 it is shown that equality is an equivalence relation. When it is necessary to refer to $\alpha$-equivalence, as opposed to the framework's equality, the former will be denoted $\equiv$.

The main advantage of this concrete definition of equality is that it is syntax directed. The sequence of rules to be applied is determined by the syntax of the term on the left, and the instantiation of the metavariables in the rule schemas is determined up to finitely many possibilities (in considering how to decompose an expression $E$ on the right into $\epsilon\left[E^{\prime}\right]$ ). Hence it is manifestly decidable and lends itself to interpretation as an algorithm.

\subsection{The type system of CLF}

We present first the type system of the LF fragment of CLF, then extend it to the LLF fragment and finally the full language. Before presenting the typing judgments, it is necessary to introduce the notions of signature and context, which record assumptions about the types (or kinds) of constants and variables respectively.

Definition 6 (Signatures And contexts, LF FRAgment)

$$
\begin{aligned}
& \Sigma::=\cdot \Sigma, a: K \mid \Sigma, c: A \quad \text { Signatures } \\
& \Gamma::=\cdot \Gamma, x: A \quad \text { Unrestricted contexts }
\end{aligned}
$$


It is an invariant that variables added to signatures and contexts must be unique, and the metavariables for contexts always denote contexts with unique variables.

There is a typing judgment for each syntactic category, as well as validity judgments for contexts $\Gamma$ and signatures $\Sigma$. Each of these judgments is defined in a completely syntaxdirected manner, so termination and decidability of typing is clear. For normal syntactic categories $(N, A, K)$ the operational interpretation of the type-checking judgment is that a putative type is provided, and the judgment holds if the term can be typed with the given type. In particular, a normal term such as $\lambda x . x$ may have several different types. This stands in contrast to the typical presentation of LF, where type labels are used in abstractions to ensure that every term has a unique type. For the atomic syntactic categories $(R, P)$ the situation is different: the operational meaning of the typing judgment is that it defines a partial function from an atomic term (in a given context and signature) to its unique type. The direction of the arrow $\Leftarrow$ or $\Rightarrow$ serves as a mnemonic for whether a type is being checked against or inferred, respectively.

In all cases the typing judgment is not considered to have any particular meaning unless the context and signature referred to in the judgment are valid. For the normal syntactic categories, the typing judgment is meaningless unless the type referred to in the judgment is valid as well. For the atomic syntactic categories, it will be proved that whenever a typing is derivable and the context and signature mentioned in the typing are valid, the type mentioned in the judgment is valid. The signature $\Sigma$ subscripting the various judgments is often omitted - it is invariant in the course of a typing derivation.

DEFINITION 7 (TYPING, LF FRAGMENT)

$\vdash \Sigma$ ok

[Signature validity]

$$
\overline{\vdash \cdot \mathrm{ok}} \quad \frac{\vdash \Sigma \mathrm{ok} \cdot \vdash_{\Sigma} K \Leftarrow \mathrm{kind}}{\vdash \Sigma, a: K \text { ok }} \quad \frac{\vdash \Sigma \mathrm{ok} \cdot \vdash_{\Sigma} A \Leftarrow \mathrm{type}}{\vdash \Sigma, c: A \text { ok }}
$$

$\vdash_{\Sigma} \Gamma$ ok

[Context validity]

$$
\overline{\vdash_{\Sigma} \cdot \mathrm{ok}} \quad \frac{\vdash_{\Sigma} \Gamma \text { ok } \Gamma \vdash_{\Sigma} A \Leftarrow \mathrm{type}}{\vdash_{\Sigma} \Gamma, x: A \text { ok }}
$$

$\Gamma \vdash_{\Sigma} K \Leftarrow$ kind

[Kind checking]

$$
\overline{\Gamma \vdash \text { type } \Leftarrow \text { kind }} \text { typeKF } \quad \frac{\Gamma \vdash A \Leftarrow \text { type } \Gamma, x: A \vdash K \Leftarrow \text { kind }}{\Gamma \vdash \Pi x: A . K \Leftarrow \text { kind }} \Pi \mathbf{K F}
$$

$\Gamma \vdash_{\Sigma} A \Leftarrow$ type

[Type checking]

$$
\frac{\Gamma \vdash A \Leftarrow \text { type } \quad \Gamma, x: A \vdash B \Leftarrow \text { type }}{\Gamma \vdash \Pi x: A . B \Leftarrow \text { type }} \Pi \mathbf{F} \quad \frac{\Gamma \vdash P \Rightarrow \text { type }}{\Gamma \vdash P \Leftarrow \text { type }} \Rightarrow \text { type } \Leftarrow
$$

$\Gamma \vdash_{\Sigma} P \Rightarrow K$

[Atomic type constructor inference]

$$
\frac{\Gamma \vdash P \Rightarrow \Pi x: A . K \quad \Gamma \vdash N \Leftarrow A}{\Gamma \vdash a \Rightarrow \Sigma(a)} a \quad \frac{\Gamma \vdash E}{\Gamma \vdash P N \Rightarrow \text { inst_k }_{A}(x . K, N)} \Pi \mathbf{K E}
$$

$\Gamma \vdash_{\Sigma} N \Leftarrow A$

[Normal object checking]

$$
\frac{\Gamma, x: A \vdash N \Leftarrow B}{\Gamma \vdash \lambda x . N \Leftarrow \Pi x: A . B} \text { ПI } \quad \frac{\Gamma \vdash R \Rightarrow P^{\prime} \quad P^{\prime}=P}{\Gamma \vdash R \Leftarrow P} \Rightarrow \Leftarrow
$$


$\Gamma \vdash_{\Sigma} R \Rightarrow A$

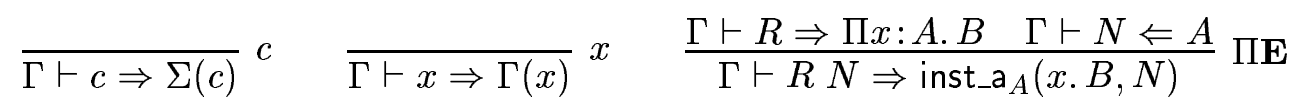

The typing rules $[\Pi \mathbf{K E}]$ and $[\Pi \mathbf{E}]$ involve the operation of instantiating a variable in a dependent type (or kind) with an object. In the first-order case, an ordinary captureavoiding substitution will suffice. However, since $\beta$-redices are not syntactically allowed in CLF, at higher type some computation must occur in order to find the term corresponding to the result of the instantiation. This is achieved by the instantiation operator inst_a ${ }_{A}(x . B, N)$, which computes the result of instantiating the variable $x$ in the type $B$ with the object $N$. The instantiation operator is indexed by the type $A$ of the object being substituted. In the first-order case, we have that inst_a $a_{\mathrm{a}}(x . B, N) \equiv[N / x] B$, but at higher type, more complex situations arise:

$$
\text { inst_a }_{\mathrm{a} \rightarrow \mathrm{a}}(x \cdot \mathrm{b}(\lambda y \cdot \mathrm{c}(x(x y))), \lambda z \cdot \mathrm{d} z) \equiv \mathrm{b}(\lambda y \cdot \mathrm{c}(\mathrm{d}(\mathrm{d} y)))
$$

The instantiation operators for each syntactic category are defined in Section 4.1.

In contrast to the usual presentation of LF typing, there are no type conversion rules (which would fail to be syntax directed). Instead, there is an appeal to the equality judgment in the rule $[\Rightarrow \Leftarrow]$ for the coercion from atomic objects to normal objects. In Section 4.5 type conversion for the typing judgments for normal syntactic categories is shown to be admissible.

In order to extend the type system to the LLF fragment, we introduce a new context for linear hypotheses. We depart slightly from the concrete syntax in adhering to the usual convention that a metavariable like $\Delta$ denotes an equivalence class of linear contexts up to rearrangement. Note that there is no issue of dependency, as there would be in rearranging an unrestricted context $\Gamma$, because types cannot depend on linear variables.

\section{DeFinition 8 (CONTEXts, LLF FRAGMENT)}

$$
\Delta::=\cdot \Delta, x \uparrow A \quad \text { Linear contexts }
$$

The typing judgments for objects must be modified in order to account for linear hypotheses - the new formulation depends on a pair of contexts $\Gamma ; \Delta$ in the style of dual intuitionistic linear logic [Bar96]. The following definition includes all the inference rules from the LF fragment that have to be revised for this reason. Note that type constructors and kinds never depend on linear variables.

DeFinition 9 (TYPing, LLF FRAGMENT)

$\Gamma \vdash_{\Sigma} \Delta$ ok

[Linear context validity]

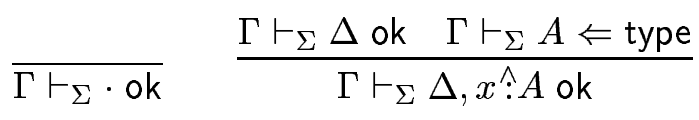

$\Gamma \vdash_{\Sigma} A \Leftarrow$ type

$$
\begin{gathered}
\frac{\Gamma \vdash A \Leftarrow \text { type } \quad \Gamma \vdash B \Leftarrow \text { type }}{\Gamma \vdash A \multimap B \Leftarrow \text { type }} \multimap \mathbf{F} \\
\frac{\Gamma \vdash A \Leftarrow \text { type } \Gamma \vdash B \Leftarrow \text { type }}{\Gamma \vdash A \& B \Leftarrow \text { type }} \& \mathbf{F} \quad \frac{}{\Gamma \vdash \top \Leftarrow \text { type }} \mathrm{T}
\end{gathered}
$$

[Type checking, extended] 
$\Gamma \vdash_{\Sigma} P \Rightarrow K$

[Atomic type inference, revised]

$$
\frac{\Gamma \vdash P \Rightarrow \Pi x: A . K \quad \Gamma ; \cdot \vdash N \Leftarrow A}{\Gamma \vdash P N \Rightarrow \text { inst_k } k_{A}(x . K, N)} \Pi \mathbf{K E}
$$

$\Gamma ; \Delta \vdash_{\Sigma} N \Leftarrow A$

[Normal object checking, revised and extended]

$$
\begin{gathered}
\frac{\Gamma, x: A ; \Delta \vdash N \Leftarrow B}{\Gamma ; \Delta \vdash \lambda x . N \Leftarrow \Pi x: A . B} \text { ПI } \quad \frac{\Gamma ; \Delta \vdash R \Rightarrow P^{\prime} \quad P^{\prime}=P}{\Gamma ; \Delta \vdash R \Leftarrow P} \Rightarrow \Leftarrow \\
\frac{\Gamma ; \Delta, x \wedge A \vdash N \Leftarrow B}{\Gamma ; \Delta \vdash \hat{\lambda} x . N \Leftarrow A \multimap B} \multimap \mathbf{I} \\
\frac{\Gamma ; \Delta \vdash N_{1} \Leftarrow A \quad \Gamma ; \Delta \vdash N_{2} \Leftarrow B}{\Gamma ; \Delta \vdash\left\langle N_{1}, N_{2}\right\rangle \Leftarrow A \& B} \& \mathbf{I} \quad \frac{\overline{\Gamma ; \Delta \vdash\langle\rangle \Leftarrow \top}}{T} \mathbf{I}
\end{gathered}
$$

$\Gamma ; \Delta \vdash_{\Sigma} R \Rightarrow A$

[Atomic object inference, revised and extended]

$$
\begin{aligned}
& \overline{\Gamma ; \cdot \vdash c \Rightarrow \Sigma(c)}^{c} \quad \overline{\Gamma ; \cdot \vdash x \Rightarrow \Gamma(x)}^{x} \quad \frac{\Gamma ; \Delta \vdash R \Rightarrow \Pi x: A . B \quad \Gamma ; \cdot \vdash N \Leftarrow A}{\Gamma ; \Delta \vdash R N \Rightarrow \text { inst_a }_{A}(x . B, N)} \Pi \mathbf{E} \\
& \frac{\Gamma ; \Delta_{1} \vdash R \Rightarrow A \multimap B \quad \Gamma ; \Delta_{2} \vdash N \Leftarrow A}{\Gamma ; x \uparrow A \vdash x \Rightarrow A}-\mathbf{E} \\
& \frac{\Gamma ; \Delta \vdash R \Rightarrow A \& B}{\Gamma ; \Delta \vdash \pi_{1} R \Rightarrow A} \& \mathbf{E}_{1} \quad \frac{\Gamma ; \Delta \vdash R \Rightarrow A \& B}{\Gamma ; \Delta \vdash \pi_{2} R \Rightarrow B} \& \mathbf{E}_{2}
\end{aligned}
$$

Finally, we are ready to extend the type system to the full CLF language. This requires one more kind of context to record the types of patterns.

Definition 10 (Contexts, Full CLF)

$$
\Psi::=\cdot \mid p \bumpeq S, \Psi \quad \text { Pattern contexts }
$$

There are additional judgments for the validity of pattern contexts and synchronous types, and for typing expressions and monadic objects. Note that pattern contexts $\Psi$ are ordered but do not allow dependencies.

Definition 11 (TyPing, FUll CLF)

$\Gamma \vdash_{\Sigma} \Psi$ ok

[Pattern context validity]

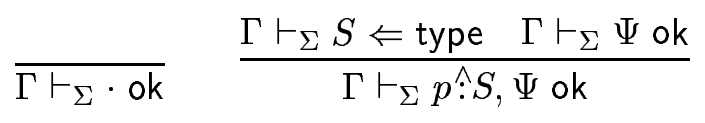

$\Gamma \vdash_{\Sigma} A \Leftarrow$ type

[Type checking, extended]

$$
\frac{\Gamma \vdash S \Leftarrow \text { type }}{\Gamma \vdash\{S\} \Leftarrow \text { type }}\{\} \mathbf{F}
$$

$\Gamma \vdash_{\Sigma} S \Leftarrow$ type

[Synchronous type checking]

$$
\begin{gathered}
\frac{\Gamma \vdash S_{1} \Leftarrow \text { type } \quad \Gamma \vdash S_{2} \Leftarrow \text { type }}{\Gamma \vdash S_{1} \otimes S_{2} \Leftarrow \text { type }} \otimes \mathbf{F} \quad \overline{\Gamma \vdash 1 \Leftarrow \text { type }} 1 \mathbf{F} \\
\frac{\Gamma \vdash A \Leftarrow \text { type } \quad \Gamma, x: A \vdash S \Leftarrow \text { type }}{\Gamma \vdash \exists x: A . S \Leftarrow \text { type }} \exists \mathbf{F}
\end{gathered}
$$


$\Gamma ; \Delta \vdash_{\Sigma} N \Leftarrow A$

[Normal object checking, extended]

$$
\frac{\Gamma ; \Delta \vdash E \leftarrow S}{\Gamma ; \Delta \vdash\{E\} \Leftarrow\{S\}}\{\} \mathbf{I}
$$

$\Gamma ; \Delta \vdash_{\Sigma} E \leftarrow S$

[Expression checking]

$$
\frac{\Gamma ; \Delta_{1} \vdash R \Rightarrow\left\{S_{0}\right\} \quad \Gamma ; \Delta_{2} ; p \wedge S_{0} \vdash E \leftarrow S}{\Gamma ; \Delta_{1}, \Delta_{2} \vdash(\text { let }\{p\}=R \text { in } E) \leftarrow S}\{\} \mathbf{E}
$$

$\frac{\Gamma ; \Delta \vdash M \Leftarrow S}{\Gamma ; \Delta \vdash M \leftarrow S} \Leftarrow \leftarrow$

$\Gamma ; \Delta ; \Psi \vdash_{\Sigma} E \leftarrow S$

[Pattern expansion]

$$
\begin{aligned}
& \frac{\Gamma ; \Delta \vdash E \leftarrow S}{\Gamma ; \Delta ; \cdot \vdash E \leftarrow S} \leftarrow \leftarrow
\end{aligned}
$$

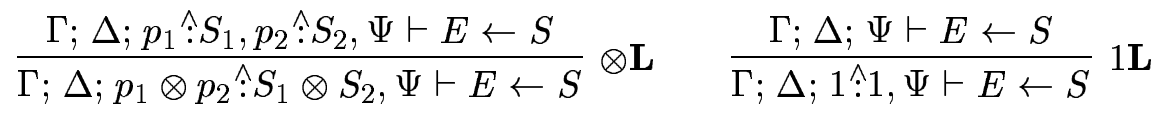

$$
\begin{aligned}
& \frac{\Gamma, x: A ; \Delta ; p \wedge S_{0}, \Psi \vdash E \leftarrow S}{\Gamma ; \Delta ;[x, p]: \exists x: A . S_{0}, \Psi \vdash E \leftarrow S} \exists \mathbf{L} \quad \frac{\Gamma ; \Delta, x \wedge A ; \Psi \vdash E \leftarrow S}{\Gamma ; \Delta ; x \wedge A, \Psi \vdash E \leftarrow S} \text { AL }
\end{aligned}
$$

$\Gamma ; \Delta \vdash_{\Sigma} M \Leftarrow S$

[Monadic object checking]

$$
\begin{gathered}
\frac{\Gamma ; \Delta_{1} \vdash M_{1} \Leftarrow S_{1} \quad \Gamma ; \Delta_{2} \vdash M_{2} \Leftarrow S_{2}}{\Gamma ; \Delta_{1}, \Delta_{2} \vdash M_{1} \otimes M_{2} \Leftarrow S_{1} \otimes S_{2}} \otimes \mathbf{I} \quad \overline{\Gamma ; \cdot \vdash 1 \Leftarrow 1} 1 \mathbf{I} \\
\frac{\Gamma ; \vdash N \Leftarrow A \quad \Gamma ; \Delta \vdash M \Leftarrow \text { inst_s }_{A}(x . S, N)}{\Gamma ; \Delta \vdash[N, M] \Leftarrow \exists x: A . S} \exists \mathbf{I}
\end{gathered}
$$

The rule $[\exists \mathbf{I}]$ requires another kind of instantiation operator in order to instantiate the dependent variable in a dependent pair type.

A summary of all the judgments and rules of CLF can be found in Appendix A.

\subsection{Related work}

As noted above, CLF includes the LF and LLF frameworks as fragments, where the CLF counterpart of an LF or LLF object is its canonical form with type labels omitted. The CLF extension is conservative; anyone who knows how to use LF or LLF can bring their (canonical) specifications to CLF and use them without modification. There is a further "modularity" property: given an LF or LLF signature $\Sigma_{1}$ and a disjoint CLF signature $\Sigma_{2}$, both of which are valid, and a type $A$ well-formed in $\Sigma_{1}$, the set of objects of type $A$ in $\Sigma_{1}, \Sigma_{2}$ is the same as the set of objects of type $A$ in $\Sigma_{1}$ alone. $^{1}$

The LLF framework was motivated as the largest fragment of intuitionistic linear logic having a proof term assignment without commuting conversions [Cer96]. The equational theory that would be associated with commuting conversions was seen as intractable. Pfenning and Davies note that the commuting conversions of Moggi's monadic metalanguage [Mog89, Mog91] can be eliminated by creating a new typing judgment associated with a new class of object, the expressions [PD01]. They exhibit a compositional translation from the monadic metalanguage into their proof term assignment for lax logic [FM97].

\footnotetext{
${ }^{1}$ The modularity result does not hold for arbitrary disjoint CLF signatures $\Sigma_{1}$ and $\Sigma_{2}$. It could be recovered by replacing the monadic type constructor with a countably infinite family of "tagged" monadic type constructors and requiring that the sets of tags mentioned in $\Sigma_{1}$ and $\Sigma_{2}$ be disjoint.
} 
The translation takes monadic metalanguage terms related by commuting conversions into identical terms of the target language. CLF exploits their idea to eliminate commuting conversions that would otherwise be associated with the monadic type and the synchronous types. The monadic type constructor $\{S\}$ would be written $\bigcirc S$ in lax logic.

Further remarks on how CLF relates to frameworks outside the LF family appear at the end of the following section.

\section{Toward a methodology for representing concurrent sys- tems}

The following are a few remarks on the methodology associated with the CLF language. For further details, the reader may consult the companion report on applications [CPWW02].

A logical framework in the LF tradition is not only a type theory. It is also a methodology for representing the deductive systems of interest within that type theory [Pfe99]. The LF methodology represents object-language judgments by LF types and object-language deductions by LF objects. Other syntactic entities of the object language (propositions, expressions, types, etc.) are also represented by LF objects. In each case an adequacy theorem establishes that there is a compositional bijection between well-formed entities of the object language and well-typed canonical objects in LF (of a certain type). The higher types and dependent types available in LF assist in this task: LF abstraction models objectlanguage binding, $\alpha$-conversion, and substitution ("higher-order abstract syntax" [PE88]); $\mathrm{LF}$ abstraction also models hypothetical and parametric judgments of the object language; and LF dependent function types enforce well-formedness constraints on object-language deductions.

For this reason, LF representations do not need to deal explicitly with matters of variable binding and $\alpha$-conversion, and certain kinds of errors in specification associated with such binding constructs are impossible to make. Similarly, LF representations do not need to explicitly encode the relation between an object-language deduction and the proposition that it proves - the proposition that a deduction proves becomes an intrinsic part of its (dependent) LF type. In this way, proof checking reduces to LF type checking, which is decidable and efficient.

Linear LF seeks to expand these benefits to the case of linear hypothetical judgments. Linear hypotheses make mutable state an intrinsic part of the framework. When representing an object system involving mutable state, it is not necessary to represent the state as an explicit object, nor to explicitly specify operations for adding information to the state, changing it, or withdrawing information from it. This idea takes on a particularly simple form when the state consists of the presence or absence of any of a set of discrete resources, as in the following example.

\subsection{An example: Petri nets with labeled tokens}

Here we consider a simple example of an object system involving state: a Petri net [Pet62] with labeled tokens. Such a Petri net consists of a directed bipartite graph of places and transitions. A state of the net consists of a mapping from a finite set of tokens, each uniquely labeled, to the places. ${ }^{2}$ A computation step consists of choosing a transition, removing a

\footnotetext{
${ }^{2}$ One requirement of the LLF representation methodology, when applied to systems involving discrete sets of resources, is that the resources be distinguishable. Hence the tokens of the Petri net must carry
} 


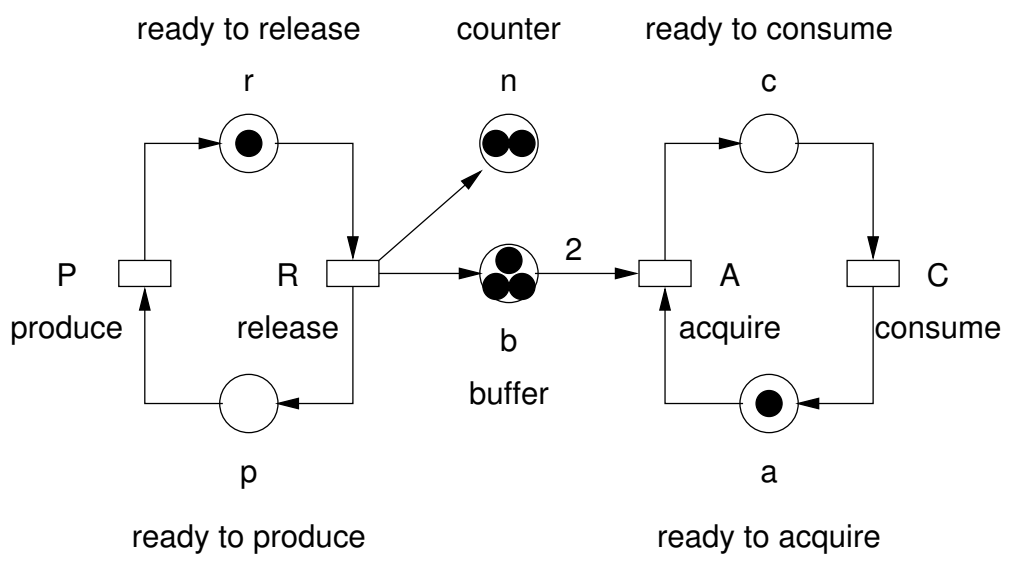

Figure 1: An example Petri net

token from each of its antecedent places, and adding a fresh token to each of its succedent places. A transition cannot be chosen if any of its antecedent places has no tokens.

For example, Figure 1 shows a labeled Petri net with six places and four transitions. Our simple representation of the net in the LLF fragment of CLF models the places by LLF type constants and the transitions and tokens by LLF objects. Each token has a type corresponding to the place in which it is located. (A more sophisticated representation might model places as objects and introduce a two-place judgment "token $x$ is in place $y . ")$ The transitions are linear functions in continuation-passing style, "consuming" linear hypotheses associated with the antecedent places and introducing new linear hypotheses associated with the succedent places. A type G represents the continuation. Thus we have the following signature:

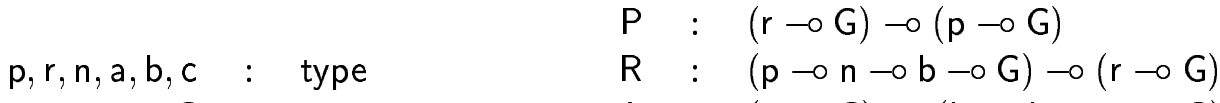

$$
\begin{aligned}
& G \quad: \text { type } \quad A:(c \multimap G) \multimap(b \multimap b \multimap a \multimap G) \\
& C:(a \multimap G) \multimap(c \multimap G)
\end{aligned}
$$

The adequacy theorem for this representation states:

Final state $q_{1}, \ldots, q_{n}$ can be reached from initial state $p_{1}, \ldots, p_{m}$ iff there is an object $N$ such that

$$
\cdot ; \vdash N \Leftarrow\left(q_{1} \multimap \ldots \multimap q_{n} \multimap \mathrm{G}\right) \multimap\left(p_{1} \multimap \ldots \multimap p_{m} \multimap \mathrm{G}\right)
$$

Moreover, there is a bijection between sequences of firings of the transition rules of the Petri net and such canonical objects.

Two examples of such objects are as follows. The first represents the firing of $\mathrm{R}$ following by the firing of $\mathrm{A}$ in the shown initial state. The second shows the same firings in the opposite order. Here the abbreviation $\hat{\lambda} x_{1}, x_{2}, \ldots, x_{n} . N$ stands for a curried sequence of linear $\lambda$-abstractions. The outermost $\lambda$-abstractions have been elided.

unique labels. It is possible that proof irrelevance [Pfe01a] could offer a way of modeling indistinguishability. 


$$
\begin{aligned}
& \Delta=f \hat{A}(\mathrm{c} \multimap \mathrm{b} \multimap \mathrm{b} \multimap \mathrm{n} \multimap \mathrm{n} \multimap \mathrm{n} \multimap \mathrm{p} \multimap \mathrm{G}), \\
& r_{1} \wedge \mathrm{r}, n_{1} \wedge \mathrm{n}, n_{2} \wedge \mathrm{n}, b_{1} \wedge \mathrm{b}, b_{2} \wedge \mathrm{b}, b_{3} \wedge \mathrm{b}, a_{1} \wedge \mathrm{a} \\
& ; \Delta \vdash \mathrm{R}^{\wedge}\left(\hat{\lambda} p_{1}, n_{3}, b_{4} \cdot \mathrm{A}^{\wedge}\left(\hat{\lambda} c_{1} \cdot f^{\wedge} c_{1} \wedge b_{3}{ }^{\wedge} b_{4} \wedge n_{1} \wedge n_{2} \wedge n_{3} \wedge p_{1}\right)^{\wedge} b_{1} \wedge b_{2} \wedge a_{1}\right)^{\wedge} r_{1} \Leftarrow \mathrm{G} \\
& ; \Delta \vdash \mathrm{A}^{\wedge}\left(\hat{\lambda} c_{1} \cdot \mathrm{R}^{\wedge}\left(\hat{\lambda} p_{1}, n_{3}, b_{4} \cdot f^{\wedge} c_{1} \wedge b_{3}{ }^{\wedge} b_{4} \wedge n_{1} \wedge n_{2} \wedge n_{3} \wedge p_{1}\right)^{\wedge} r_{1}\right)^{\wedge} b_{1} \wedge b_{2} \wedge a_{1} \Leftarrow \mathrm{G}
\end{aligned}
$$

The benefit of the LLF methodology is clear: it is not necessary to explicitly manage a "list" of tokens and axiomatize operations for adding and removing tokens from the list, as one would have to do in LF. In LF one would also have to prove various interaction laws for the token-list-managing operations; in LLF, the required principles are proved once and for all as structural laws of the framework's linear hypothetical judgment. However, there is also room for improvement. We might have hoped for an adequacy theorem relating LLF objects to concurrent computations of the Petri net, that is, equivalence classes of computations under rearragement of independent steps. But this strengthened adequacy theorem does not hold. For example, the two LLF terms above correspond to computations differing only in the order of independent $\mathrm{R}$ and A steps: no labeled token indexing the $\mathrm{R}$ step is involved in the A step, and vice versa. But the structure of the LLF representation nonetheless requires that the two orderings be represented by different terms. In essence, the continuationpassing style of the representation forces a sequentialization of the computation.

\subsection{Representing LPNs in CLF}

It is tempting to think that this issue can be solved by adding more connectives to the framework. Why not work with a framework containing a full complement of linear logical operators (including $1, A \otimes B, ! A$ ) and replace

$$
c_{k}:\left(q_{1} \multimap \ldots \multimap q_{n} \multimap \mathrm{G}\right) \multimap\left(p_{1} \multimap \ldots \multimap p_{m} \multimap \mathrm{G}\right)
$$

with the apparently more straightforward

$$
c_{k}^{\prime}: p_{1} \otimes \ldots \otimes p_{m} \multimap q_{1} \otimes \ldots \otimes q_{n} ?
$$

Unfortunately, such an extension would not be conservative over the LF and LLF fragments of the type language. In fact, it would have a catastrophic effect on adequacy results for even very simple LF encodings. Consider a simple LF representation of the natural numbers together with an additional constant $c$ of multiplicative unit type:

$$
\begin{aligned}
& \text { nat : type } \quad s: \text { nat } \rightarrow \text { nat } \\
& \mathrm{z} \text { : nat } \mathrm{c}: 1
\end{aligned}
$$

The problem is that terms such as (let $1=\mathrm{c}$ in $\mathrm{z}$ : nat) destroy the bijective correspondence of the type nat with the set of natural numbers. ${ }^{3}$ Similar examples would arise in the presence of a constant of type $A \otimes B, ! A, A \oplus B$, or 0 . So the adequacy of the LF encoding is destroyed by the presence of even a single object constant having a type given by one of the new type constructors.

The underlying problem is that the destructors associated with these synchronous types involve "polymorphic" binding constructs that do not constrain the type of the object

\footnotetext{
${ }^{3}$ Examples such as $(\hat{\lambda} x$. let $1=x$ in z: $1 \multimap$ nat $)$ show that the term above cannot simply be equal to $z$.
} 
resulting from the binding. CLF's monadic type extracts us from this difficulty by restricting the awkward binding constructs to the monad. This encapsulation protects the pure LF and LLF fragments of CLF from the new constructs. All encodings already devised for LF or LLF remain adequate, and their adequacy proofs can remain exactly the same. Furthermore, as has already been noted, the separation between expressions and object rules out commuting conversions, simplifying the equational theory.

Using CLF's multiplicative conjunction $\otimes$, our transition rule can be rewritten as

$$
c_{k}^{\prime \prime}: p_{1} \multimap \ldots \multimap p_{m} \multimap\left\{q_{1} \otimes \ldots \otimes q_{n}\right\}
$$

where currying eliminates the use of $\otimes$ on the left-hand side. The multiplicative unit 1 covers the case $n=0$. Though it does not arise here, a modified model of Petri nets might also have transitions generating elements of persistent (unrestricted) type. In that case we would have exponentials ! $q$ on the right as well. (Exponentials on the left can be curried away using the unrestricted function type.)

As an aside, while one might think that the presence of the exponential type constructor ! would render the intuitionistic function type $A \rightarrow B$ definable by $\{! A\} \multimap B$, this is not in fact the case. As a simple counterexample we may see that while $a \rightarrow a$ ( $a$ being an uninhabited atomic type) is inhabited by $\lambda x . x$, the type $\{! a\} \multimap a$ is not inhabited. The type $\{! a\} \multimap\{a\}$ is inhabited by $\hat{\lambda} x$. $\{$ let $\{! y\}=x$ in $y\}$, however.

Thus, in CLF, the Petri net example is represented almost as in intuitionistic linear logic, except that the right-hand sides of the linear implications use the monad.

$$
\begin{aligned}
& P: \quad p \multimap\{r\} \\
& R \quad: \quad r \multimap\{p \otimes n \otimes b\} \\
& A:: \quad b \multimap b \multimap a \multimap\{c\} \\
& C \quad: \quad c \multimap\{a\}
\end{aligned}
$$

The two example Petri net executions shown above in the LLF encoding correspond to the following objects in the new encoding:

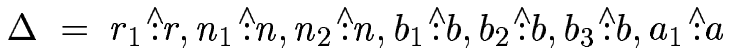

$$
\begin{aligned}
& S=c \otimes b \otimes b \otimes n \otimes n \otimes n \otimes p \\
& \cdot ; \Delta \vdash\left\{\text { let }\left\{p_{1} \otimes n_{3} \otimes b_{4}\right\}=R^{\wedge} r_{1} \text { in let }\left\{c_{1}\right\}=A^{\wedge} b_{1} \wedge b_{2} \wedge a_{1}\right. \text { in } \\
& \left.c_{1} \otimes b_{3} \otimes b_{4} \otimes n_{1} \otimes n_{2} \otimes n_{3} \otimes p_{1}\right\} \Leftarrow\{S\} \\
& \cdot ; \Delta \vdash\left\{\text { let }\left\{c_{1}\right\}=A^{\wedge} b_{1}^{\wedge} b_{2}^{\wedge} a_{1} \text { in let }\left\{p_{1} \otimes n_{3} \otimes b_{4}\right\}=R^{\wedge} r_{1}\right. \text { in } \\
& \left.c_{1} \otimes b_{3} \otimes b_{4} \otimes n_{1} \otimes n_{2} \otimes n_{3} \otimes p_{1}\right\} \Leftarrow\{S\}
\end{aligned}
$$

It is now easy to see that the two executions are equal. This idea is crystallized as an improved adequacy theorem:

Final state $q_{1}, \ldots, q_{n}$ can be reached from initial state $p_{1}, \ldots, p_{m}$ iff there is a object $N$ such that

$$
; \cdot \vdash N \Leftarrow p_{1} \multimap \ldots \multimap p_{m} \multimap\left\{q_{1} \otimes \ldots \otimes q_{n}\right\}
$$

Moreover, there is a bijection between concurrent executions of the transition rules of the Petri net and equivalence classes of such objects modulo $=$. 
While at first this may seem a minor modification, it has far-reaching consequences. Experience with logical frameworks has shown many times that natural encodings lead to deeper understanding of the underlying logical and computational principles, while contrived encodings often do not shed much light on the subject of study. These advantages are multiplied when considering algorithms for manipulating the representations, for proof search, and for meta-theoretic reasoning, because the principles embodied in and provided by the framework have been factored out and do not need to be re-implemented for each encoding.

The representation principle for CLF, then, can be summarized by "concurrent computations as monadic expressions." Each computation step in a concurrent computation becomes a binding let $\{p\}=R$ in $E$ possibly "consuming" some linear hypotheses in $R$ and "producing" more linear hypotheses in $p$, which will be available in the rest of the computation $E$.

While there is not space here to discuss more interesting examples of CLF representations, the companion technical report [CPWW02] contains examples including a fuller discussion of Petri net encodings; synchronous and asynchronous $\pi$-calculi; an ML-like language with references and concurrency in the style of CML; and the security protocol language MSR.

\subsection{Related work}

The LF representation methodology is discussed in detail in the handbook article on logical frameworks by Pfenning [Pfe99]. Further examples of CLF representations, including a full development of the Petri net example, are in the technical report by Cervesato et al. [CPWW02]. The idea of representing Petri nets by linear hypothetical judgments has a long history, going back to Martí-Oliet and Meseguer [MOM91].

Monadic encapsulation in the context of functional programming and type theory begins with Moggi's monadic metalanguage [Mog89, Mog91]. Prawitz describes a proof theory for modal logics [Pra65], from which Moggi's presentation of the monadic metalanguage inherits. Pfenning and Davies [PD01] revisit the question of proof theory for modal logic, reinterpreting it on the basis of a judgmental approach in the style promulgated by MartinLöf [ML96]. Their approach improves on the original formulation of the monadic metalanguage in that commuting conversions are no longer needed. Their judgmental analysis of the modal operator of lax logic is the basis for CLF's modal type constructor and the associated CLF judgments. They do not, however, consider the existence of canonical forms, and their proof term language, unlike CLF, relies on $\beta \eta$-conversion.

There have been many other formalisms proposed for the representation of concurrent systems, many having elements in common with the CLF approach. Abramsky's "proofsas-processes" relates classical linear logic with the synchronous $\pi$-calculus [Abr93, BS94]. Here concurrent computation corresponds to proof normalization (cut elimination), giving the system a functional flavor. Concurrent computations (traces) are thus not reified as objects, as they are in CLF.

Closer to the CLF view are approaches in which logical formulas are identified with processes and proofs with concurrent computations. Thus, these are nearer to logic programming in the sense of proof search [MNPS91] than to functional programming. For example, Miller outlines a translation from the $\pi$-calculus into linear logic: processes become LL propositions and $\pi$-calculus reduction becomes LL entailment [Mil92]. These ideas 
are generalized and reformulated as a logical framework in Miller's proposal for the specification logic Forum [Mil96, Chi95]. Forum offers a paradigm for viewing logic programming as concurrent computation, and so it is likely that the logic programming interpretation for CLF will draw heavily from it. However, in Forum proofs cannot be manipulated as first-class objects - not even cut elimination is treated, let alone an equational theory on proofs. The same is true of LinLog [And92], LO [AP91], ACL [KY93], Lygon [HPW96], and LLP [HWTK98], all of which treat logic programming over other fragments of classical or intuitionistic linear logic.

Perrier describes how the basic idea of linear logic programming as concurrent computation can be improved by adopting proof nets [Gir87] rather than sequent calculus proofs as the fundamental computational objects [Per98]. Again, however, proof nets are treated only meta-theoretically - they are not first-class terms of the process language. The key foundational idea of Elf, that proofs should be just like any other objects of the framework, does not apply.

Barber proposes a very general type theory based on linear operators and proves decidability in the general framework [Bar97]. Since the generalization includes the DILL type theory and the action calculus as special cases, this immediately entails the decidability of the DILL type theory. However, there is no treatment of dependent types, nor would DILL be adequate for LF-style representations for the reasons outlined in Section 3.2. Also, the presentation does not seem to lend itself to concrete implementation as a mechanized framework. Finally, Barber's reliance on proof nets would seem to render the extension to additive sum types problematic, while for CLF it is straightforward, because the issue of commuting conversions (other than an appropriate generalization of the permutative conversions) does not arise.

Honsell et al. develop perhaps the most significant application of a logical framework in the sphere of concurrency [HMS01]. They present an encoding of the $\pi$-calculus in the Coq system, a framework based on a higher-order type theory with (co)inductive types, and work out some rather advanced meta-theory using the encoding. But this should be regarded as a tour de force, given that Coq offers no intrinsic support for reasoning about concurrency. Of course, even in CLF, abstract relations like the strong late bisimularity treated in their development would need to be treated explicitly. CLF's concurrent equality simplifies such reasoning; it does not obviate the need for it.

\section{Some meta-theoretic results}

The design of CLF is based on the idea that every syntactically well-formed term should be "canonical," and that the notion of $\beta \eta$-conversion should be eliminated, in favor of simple inductively defined instantiation operators for instantiating a variable in a term with an object. Thus, in developing the meta-theory of CLF there is no need to consider issues such as confluence and normalizability per se. Instead, we define the instantiation operators by a manifestly terminating recurrence, and we focus on the simple algebraic laws that the instantiation operators satisfy, and on their interaction with the framework's notion of equality.

Another new element of this approach is an expansion operator taking an atomic object to the corresponding normal object at higher type. This is necessary because the coercion rule $[\Rightarrow \Leftarrow]$ from atomic objects to normal objects can only be applied at base type. The expansion operator thus replaces the idea of $\eta$-conversion. 
Once the instantiation and expansion operators have been defined, and their algebraic properties characterized, it is possible to describe their relationship to the judgments for typing. It so happens that the instantiation operators witness the familiar substitution principles for typing (the transitivity of entailment, from the proof-theoretic point of view), and the expansion operator witnesses the identity principles for typing (the reflexivity of entailment).

\subsection{Instantiation}

The recurrence defining instantiation is based on the observation, exploited in cut elimination proofs on the logical side [Pfe00], but not so well known on the type theoretic side, that the canonical result of substituting one canonical term into another can be defined by induction on the type of the term being substituted. Accordingly, the instantiation operators are defined as a family parameterized over the type of the object being substituted. In the notation inst_c $c_{A}(x . X, N)$ this type $A$ appears as a subscript. Here $c$ is replaced by a mnemonic for the particular syntactic category to which the instantiation operator applies. The variable $x$ is to be considered bound within the term $X$ (of whatever category) being substituted into. The operators defined in this section should be thought of as applying to equivalence classes of concrete terms modulo $\alpha$-equivalence on bound variables.

Together with the instantiation operators, and defined by mutual recursion with them, is a reduction operator reduce $A(x . R, N)$ that computes the canonical object resulting from the instantiation of $x$ with $N$ in the case that the head variable head $(R)$ of the atomic object $R$ is $x$. Thus, roughly speaking, it corresponds to the idea of weak head reduction for systems with $\beta$-reduction. The instantiation operator inst_r ${ }_{A}(x . R, N)$, by contrast, is only defined if the head of $R$ is not $x$. Another distinguishing feature is that reduction on an atomic object yields a normal object, while instantiation on an atomic object yields an atomic object.

Finally, there is a type reduction operator treduce $_{A}(x . R)$ that computes the putative type of $R$ given that the head of $R$ is $x$ and the type of $x$ is $A .^{4}$ Type reduction is used in side conditions that ensure that the recurrence defining instantiation is well-founded.

The first definition covers the LF fragment of CLF.

Definition 12 (Instantiation, LF FRAgment)

treduce $_{A}(x . R) \equiv B$

[Type reduction]

$$
\begin{aligned}
& \text { treduce }_{A}(x . x) \equiv A \\
& \text { treduce }_{A}(x . R N) \equiv C \quad \text { if treduce }{ }_{A}(x . R) \equiv \Pi y: B . C
\end{aligned}
$$

reduce $_{A}\left(x . R, N_{0}\right) \equiv N^{\prime}$

[Reduction]

$$
\begin{aligned}
& \text { reduce }_{A}\left(x . x, N_{0}\right) \equiv N_{0} \\
& \text { reduce }_{A}\left(x . R N, N_{0}\right) \equiv \text { inst_n }_{B}\left(y . N^{\prime}, \text { inst_n }_{A}\left(x . N, N_{0}\right)\right) \\
& \quad \text { if treduce } \\
& A
\end{aligned}
$$

\footnotetext{
${ }^{4}$ Actually, to be more precise, the type of $R$ will be a substitution instance of treduce $A(x . R)$. The instantiation operators do not keep track of dependencies within the type subscript.
} 


$$
\begin{aligned}
& \text { inst_r }_{A}\left(x . R, N_{0}\right) \equiv R^{\prime} \\
& \text { [Atomic object instantiation] } \\
& \text { inst_r }_{A}\left(x . c, N_{0}\right) \equiv c \\
& \text { inst_r }{ }_{A}\left(x . y, N_{0}\right) \equiv y \text { if } y \text { is not } x \\
& \text { inst_r }_{A}\left(x . R N, N_{0}\right) \equiv\left(\text { inst_r }_{A}\left(x . R, N_{0}\right)\right)\left(\text { inst_n }_{A}\left(x . N, N_{0}\right)\right) \\
& \text { inst_n } \mathrm{n}_{A}\left(x . N, N_{0}\right) \equiv N^{\prime} \\
& \text { [Normal object instantiation] } \\
& \text { inst_n }{ }_{A}\left(x . \lambda y . N, N_{0}\right) \equiv \lambda y \text {. inst_n }{ }_{A}\left(x . N, N_{0}\right) \quad \text { if } y \notin \mathrm{FV}\left(N_{0}\right) \\
& \text { inst_n }_{A}\left(x . R, N_{0}\right) \equiv \text { inst_r }_{A}\left(x . R, N_{0}\right) \quad \text { if head }(R) \text { is not } x \\
& \text { inst_n }_{A}\left(x . R, N_{0}\right) \equiv \text { reduce }_{A}\left(x . R, N_{0}\right) \quad \text { if treduce }{ }_{A}(x . R) \equiv P
\end{aligned}
$$$$
\text { inst_p }{ }_{A}\left(x . P, N_{0}\right) \equiv P^{\prime}
$$$$
\text { inst_a } A\left(x . A, N_{0}\right) \equiv A^{\prime}
$$

[Atomic type constructor instantiation]$$
\text { inst_k } \mathrm{k}_{A}\left(x . K, N_{0}\right) \equiv K^{\prime}
$$

[Type instantiation]

[Kind instantiation]

\section{(Analogous.)}

The recurrence defining these operators is based on a structural induction. There is an outer induction on the type subscripting the operators, and an inner simultaneous induction on the two arguments. Noting first that if $\operatorname{treduce}_{A}(x . R)$ is defined, it is a subterm of $A$, the fact that the recurrence relations respect this induction order can be verified almost by inspection. The only slightly subtle case is the equation for reduce ${ }_{A}\left(x . R N, N_{0}\right)$, which is the only case in which the subscripting type changes. Here the side condition treduce $A(x . R) \equiv$ $\Pi x: B . C$ ensures that $B$ must be a strict subterm of $A$ for the reduction to be defined. An instantiation such as inst_n $\mathrm{n}_{A}(x . x x, \lambda x . x x)$ is guaranteed to fail the side condition after only finitely many expansions of the recurrence.

Another way in which an instance of the instantiation operators might fail to be defined would be if the recursive instantiation inst_r ${ }_{A}\left(x . R, N_{0}\right)$ in the same equation failed to result in a manifest lambda abstraction $\lambda y . N^{\prime}$. In fact, this could only happen if the term $N_{0}$ failed to have the ascribed type $A .{ }^{5}$ So instantiation always terminates, regardless of whether its arguments are well typed, but it is not defined in all cases. After the meta-theory is further developed, it can be shown that instantiation is always defined on well-typed terms when the types match in the appropriate way.

No substantially new issues arise in the extension to the LLF fragment.

DEFinition 13 (Instantiation, LLF FRAGMENT)

treduce $_{A}(x . R) \equiv B$

[Type reduction, extended]

$$
\begin{array}{ll}
\text { treduce }_{A}\left(x . R^{\wedge} N\right) \equiv C & \text { if treduce } \\
A & (x . R) \equiv B \multimap C \\
\text { treduce }_{A}\left(x . \pi_{1} R\right) \equiv B_{1} & \text { if treduce } \\
\text { treduce } \left._{A}(x . R) \equiv \pi_{1} \& \pi_{2} R\right) \equiv B_{2} & \text { if treduce } \\
\text { tred }_{A}(x) \equiv B_{1} \& B_{2}
\end{array}
$$

$$
\begin{aligned}
& \text { reduce }_{A}\left(x . R, N_{0}\right) \equiv N^{\prime} \\
& \text { [Reduction, extended] } \\
& \text { reduce }_{A}\left(x . R^{\wedge} N, N_{0}\right) \equiv \text { inst_n }_{B}\left(y \cdot N^{\prime}, \text { inst_n }_{A}\left(x . N, N_{0}\right)\right) \\
& \text { if treduce }{ }_{A}(x . R) \equiv B \multimap C \text { and reduce }{ }_{A}\left(x . R, N_{0}\right) \equiv \hat{\lambda} y . N^{\prime} \\
& \text { reduce }_{A}\left(x . \pi_{1} R, N_{0}\right) \equiv N_{1}^{\prime} \quad \text { if reduce } \text { red }_{A}\left(x . R, N_{0}\right) \equiv\left\langle N_{1}^{\prime}, N_{2}^{\prime}\right\rangle \\
& \text { reduce }_{A}\left(x . \pi_{2} R, N_{0}\right) \equiv N_{2}^{\prime} \quad \text { if reduce } A\left(x . R, N_{0}\right) \equiv\left\langle N_{1}^{\prime}, N_{2}^{\prime}\right\rangle
\end{aligned}
$$

\footnotetext{
${ }^{5}$ Or a substitution instance of $A$.
} 
inst_r $_{A}\left(x . R, N_{0}\right) \equiv R^{\prime}$

[Atomic object instantiation, extended]

$$
\begin{aligned}
& \text { inst_r }_{A}\left(x . R^{\wedge} N, N_{0}\right) \equiv\left(\text { inst_r }_{A}\left(x . R, N_{0}\right)\right)^{\wedge}\left(\text { inst_r }_{A}\left(x . N, N_{0}\right)\right) \\
& \text { inst_r }_{A}\left(x . \pi_{1} R, N_{0}\right) \equiv \pi_{1}\left(\text { inst_r }_{A}\left(x . R, N_{0}\right)\right) \\
& \text { inst_r }{ }_{A}\left(x . \pi_{2} R, N_{0}\right) \equiv \pi_{2}\left(\text { inst_r }{ }_{A}\left(x . R, N_{0}\right)\right)
\end{aligned}
$$

inst_n $\mathrm{n}_{A}\left(x . N, N_{0}\right) \equiv N^{\prime}$

[Normal object instantiation, extended]

$$
\begin{aligned}
& \text { inst_n }_{A}\left(x \cdot \hat{\lambda} y \cdot N, N_{0}\right) \equiv \hat{\lambda} y \cdot \text { inst_n }_{A}\left(x . N, N_{0}\right) \quad \text { if } y \notin \mathrm{FV}\left(N_{0}\right) \\
& \text { inst_n }_{A}\left(x \cdot\left\langle N_{1}, N_{2}\right\rangle, N_{0}\right) \equiv\left\langle\text { inst_n }_{A}\left(x . N_{1}, N_{0}\right), \text { inst_n }{ }_{A}\left(x . N_{2}, N_{0}\right)\right\rangle \\
& \text { inst_n } \mathrm{n}_{A}\left(x .\langle\rangle, N_{0}\right) \equiv\langle\rangle
\end{aligned}
$$

In order to extend this idea to the full CLF language, with its pattern-oriented destructor for the monadic type, it is necessary to introduce matching operators match_c $c_{S}(p . E, X)$, where $X$ is either an expression or a monadic object. The matching operator computes the result of instantiating $E$ according to the substitution on the variables of $p$ generated by matching $p$ against $X$. (The variables in $p$ should be considered bound in $E$.) In the case that $X$ is a monadic object $M_{0}$, this is straightforward: the syntax of monadic objects corresponds precisely to that of patterns. But in the case that $X$ is a let binding, an interesting issue arises:

$$
\text { match_e }{ }_{S}\left(p \text {. let }\left\{p_{1}\right\}=R_{1} \text { in } E_{1} \text {, let }\left\{p_{2}\right\}=R_{2} \text { in } E_{2}\right) \equiv \text { ? }
$$

The key is found in Pfenning and Davies' non-standard substitutions for the proof terms of the modal logics of possibility and laxity [PD01]. These analyze the structure of the object being substituted, not, as in the usual case, the term being substituted into. The effect is similar to a commuting conversion:

$$
\begin{aligned}
& \text { match_e }{ }_{S}\left(p \text {. let }\left\{p_{1}\right\}=R_{1} \text { in } E_{1} \text {, let }\left\{p_{2}\right\}=R_{2} \text { in } E_{2}\right) \equiv \\
& \quad\left(\text { let }\left\{p_{2}\right\}=R_{2} \text { in match_e } S\left(p \text {. let }\left\{p_{1}\right\}=R_{1} \text { in } E_{1}, E_{2}\right)\right)
\end{aligned}
$$

It is interesting that both non-standard substitution and pattern matching - the latter not present in Pfenning and Davies' system - rely in this way on an analysis of the object being substituted rather than the term being substituted into. In a sense, this commonality is what makes the harmonious interaction between CLF's modality and its synchronous types possible.

$$
\begin{aligned}
& \text { Definition } 14 \text { (Instantiation, FUll CLF) } \\
& \text { inst_n }{ }_{A}\left(x . N, N_{0}\right) \equiv N^{\prime} \\
& \text { [Normal object instantiation, extended] } \\
& \text { inst_n }_{A}\left(x .\{E\}, N_{0}\right) \equiv\left\{\text { inst_e }_{A}\left(x . E, N_{0}\right)\right\} \\
& \text { inst_m }_{A}\left(x . M, N_{0}\right) \equiv M^{\prime} \\
& \text { [Monadic object instantiation] } \\
& \text { inst_m }{ }_{A}\left(x . M_{1} \otimes M_{2}, N_{0}\right) \equiv \text { inst_m }_{A}\left(x . M_{1}, N_{0}\right) \otimes \text { inst_m }_{A}\left(x . M_{2}, N_{0}\right) \\
& \text { inst_m } \mathrm{m}_{A}\left(x .1, N_{0}\right) \equiv 1 \\
& \text { inst_m }_{A}\left(x .[N, M], N_{0}\right) \equiv\left[\text { inst_n } \mathrm{n}_{A}\left(x . N, N_{0}\right), \text { inst_m }{ }_{A}\left(x . M, N_{0}\right)\right] \\
& \text { inst_m }{ }_{A}\left(x . N, N_{0}\right) \equiv \text { inst_n }_{A}\left(x . N, N_{0}\right)
\end{aligned}
$$




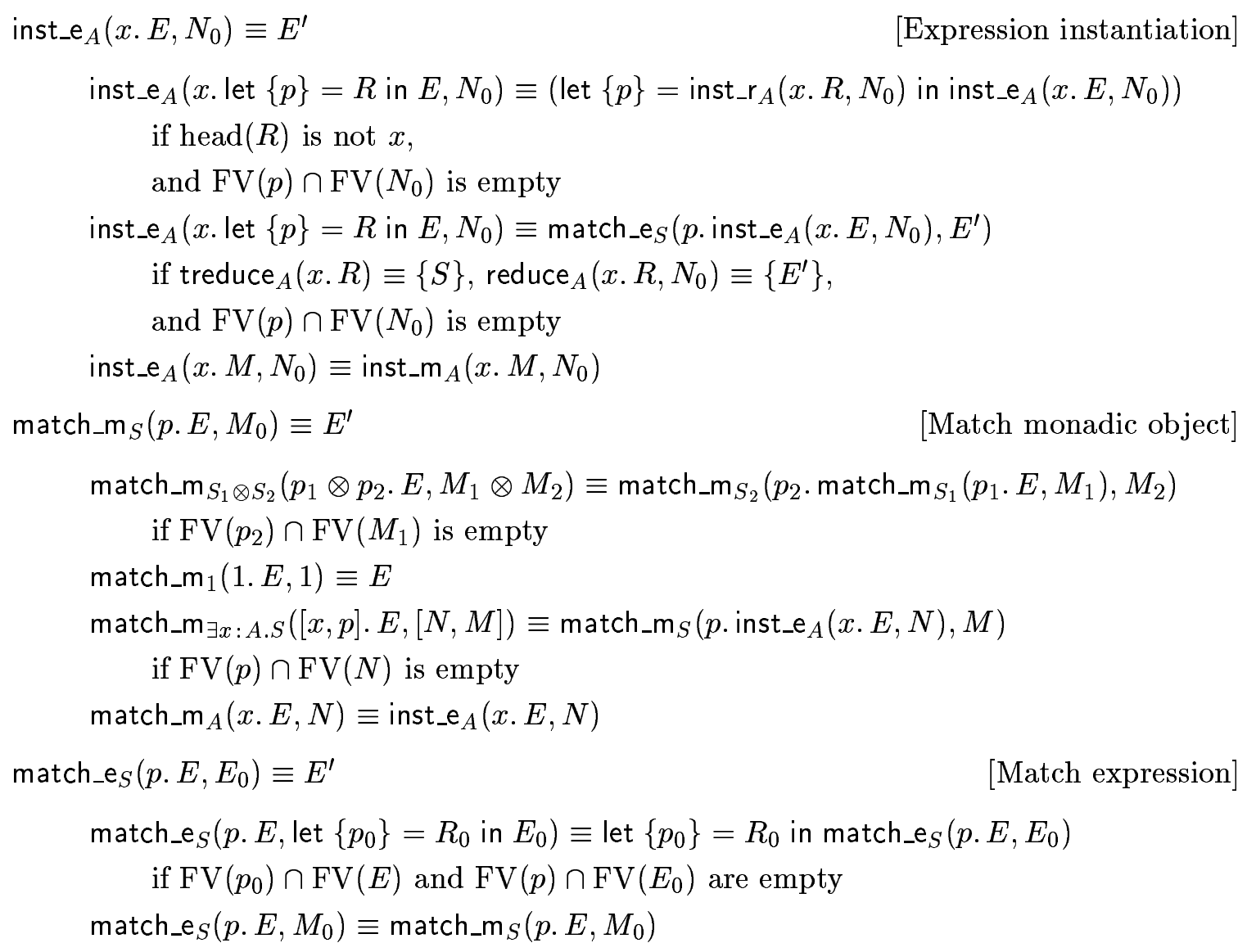

inst_s $_{A}\left(x . S, N_{0}\right) \equiv S^{\prime}$

[Synchronous type instantiation]

(Analogous.)

We interpret these recurrences as inductive definitions (adopting the least solution to the recurrence). The first theorem ensures that type reduction has been properly defined. There are also two lemmas, one of which, mentioned earlier, ensures that type reduction makes a type smaller. All are immediate by structural induction on the argument.

Theorem 1 (DEFinability of TYPE REDUCTION)

The recurrence for type reduction uniquely determines a least partial function solving the recurrence.

LEMMA 2

If treduce $_{A}(x . R)$ is defined, then head $(R)$ is $x$.

LEMMA 3

If treduce $_{A}(x . R) \equiv B$, then $B$ is a subterm of $A$.

Now we can conclude that instantiation has been properly defined.

THEOREM 4 (DEFINABILITY OF InSTANTIATION)

The recurrence for the reduction, instantiation, and matching operators uniquely determines the least partial functions (up to $\alpha$-equivalence) solving them.

Proof. The proof is by an outer structural induction on the type subscript, and an inner simultaneous structural induction on the two arguments. 


\subsection{Expansion}

The expansion operator is specified by the following equations. In some cases, new bound variables are introduced on the right-hand side of an equation. Any new variables in an instance of such an equation are required to be distinct from one another and from any other variables in the equation instance.

$$
\begin{aligned}
& \text { DEFINITION } 15 \text { (EXPANSION) } \\
& \text { expand }_{A}(R) \equiv N \\
& \text { [Expansion] } \\
& \operatorname{expand}_{P}(R) \equiv R \\
& \operatorname{expand}_{A \rightarrow B}(R) \equiv \hat{\lambda} x \text {. } \operatorname{expand}_{B}\left(R^{\wedge}\left(\operatorname{expand}_{A}(x)\right)\right) \quad \text { if } x \notin \mathrm{FV}(R) \\
& \text { expand }_{\Pi x: A . B}(R) \equiv \lambda x \text {. } \text { expand }_{B}\left(R\left(\operatorname{expand}_{A}(x)\right)\right) \quad \text { if } x \notin \mathrm{FV}(R) \\
& \text { expand }_{A \& B}(R) \equiv\left\langle\operatorname{expand}_{A}\left(\pi_{1} R\right) \text {, } \operatorname{expand}_{B}\left(\pi_{2} R\right)\right\rangle \\
& \operatorname{expand}_{\mathrm{T}}(R) \equiv\langle\rangle \\
& \operatorname{expand}_{\{S\}}(R) \equiv\left(\text { let }\{p\}=R \text { in } \operatorname{pexpand}_{S}(p)\right)
\end{aligned}
$$$$
\operatorname{pexpand}_{S}(p) \equiv M
$$

$$
\begin{aligned}
& \text { pexpand }_{S_{1} \otimes S_{2}}\left(p_{1} \otimes p_{2}\right) \equiv \operatorname{pexpand}_{S_{1}}\left(p_{1}\right) \otimes \operatorname{pexpand}_{S_{2}}\left(p_{2}\right) \\
& \text { pexpand }_{1}(1) \equiv 1
\end{aligned}
$$$$
\operatorname{pexpand}_{\exists x: A . S}([x, p]) \equiv\left[\operatorname{expand}_{A}(x), \operatorname{pexpand}_{S}(p)\right]
$$$$
\operatorname{pexpand}_{A}(x) \equiv \operatorname{expand}_{A}(x)
$$

Theorem 5 (DEFinability of EXPAnsion) 1. If $\operatorname{pexpand}_{S}\left(p_{1}\right)$ and $\operatorname{pexpand}_{S}\left(p_{2}\right)$ are both defined then $p_{1}$ and $p_{2}$ are the same up to variable renaming.

2. Given $S$, there is a pattern $p$, fresh with respect to any given set of variables, such that $\operatorname{pexpand}_{S}(p)$ is defined.

3. The recurrence for expansion uniquely determines it as a total function up to $\alpha$ equivalence.

Proof. The first part is by induction on $S$. The second and third parts are by induction on the type subscript, using the first part to ensure that the result of expand ${ }_{\{S\}}(R)$ is unique up to $\alpha$-equivalence.

\subsection{On decidability}

The next few meta-theoretic observations are on the decidability of the fundamental operators and judgments of the theory. We begin with equality. Recall that equality is totally independent of typing and is syntax directed.

Theorem 6 (Decidability of EQuality) 1. Given $R_{1}$ and $R_{2}$, it is decidable whether $R_{1}=R_{2}$.

2. Given $N_{1}$ and $N_{2}$, it is decidable whether $N_{1}=N_{2}$.

3. Given $M_{1}$ and $M_{2}$, it is decidable whether $M_{1}=M_{2}$. 
4. Given $E_{1}$ and $E_{2}$, it is decidable whether $E_{1}={ }_{\mathrm{c}} E_{2}$.

5. Given $E_{1}$ and $E_{2}$, it is decidable whether $E_{1}=E_{2}$.

6. Given $P_{1}$ and $P_{2}$, it is decidable whether $P_{1}=P_{2}$.

Proof. The proof is by simultaneous structural induction on the subjects of the judgments. The judgments have been placed in the proper order of precedence in view of the trivial coercions from $R$ to $N$ to $M$ to $E$. For example, $R_{1}=R_{2}$ is below $N_{1}=N_{2}$ only if each of $R_{1}$ and $R_{2}$ is a subterm of either $N_{1}$ or $N_{2}$, but $N_{1}=N_{2}$ is below $R_{1}=R_{2}$ only if each of $N_{1}$ and $N_{2}$ is a strict subterm of either $R_{1}$ or $R_{2}$. In the case of $E_{1}={ }_{c} E_{2}$ it suffices to note that any $E_{2}$ can be decomposed as $\epsilon\left[E_{2}^{\prime}\right]$ in only finitely many ways.

The next theorems are on the decidability of instantiation and expansion. Instantiation and expansion are both syntax directed and terminate on arbitrary terms and type subscripts. There is no requirement that the terms or type subscript be valid or that the type subscript have any particular relationship to the terms. The proofs are immediate by the same induction schemes as for the definability theorems.

THEOREM 7 (DECIDABILITY OF INSTANTIATION)

It is decidable whether any instance of the instantiation and matching operators is defined, and if so, it can be effectively computed.

Theorem 8 (DEcidability of EXPANSion) 1. Given $S$, a pattern $p$ can be effectively computed such that pexpand ${ }_{S}(p)$ is defined and can be effectively computed. The pattern $p$ can be chosen fresh with respect to any given set of variables.

2. Given $A$ and $R$, the result of expand ${ }_{A}(R)$ can be effectively computed.

The fact that instantiation and equality are decidable leads directly to the decidability of typing in the framework, since the typing rules are syntax directed and appeal only to instantiation and equality.

Lemma 9 (Unicity of INFERENCE) 1. There is a partial function typeof $(\Gamma ; \Delta \vdash R$ ) such that whenever $\Gamma ; \Delta \vdash R \Rightarrow A$ holds, $A \equiv \operatorname{typeof}(\Gamma ; \Delta \vdash R)$. Given $\Gamma, \Delta$, and $R$, it is decidable whether typeof $(\Gamma ; \Delta \vdash R)$ is defined, and if so, it can be effectively computed.

2. There is a partial function kindof $(\Gamma \vdash P)$ such that whenever $\Gamma \vdash P \Rightarrow K$ holds, $K \equiv \operatorname{kindof}(\Gamma \vdash P)$. Given $\Gamma$ and $P$, it is decidable whether $\operatorname{kindof}(\Gamma \vdash P)$ is defined, and if so, it can be effectively computed.

Proof. Immediate by the facts that typing is syntax directed, instantiation is a partial function, and instantiation is computable.

LEMMA 10

Suppose that for all $\Gamma^{\prime}$ and $\Delta^{\prime}$ it is decidable whether $\Gamma^{\prime} ; \Delta^{\prime} \vdash E \leftarrow S$. Then it is decidable whether $\Gamma ; \Delta ; \Psi \vdash E \leftarrow S$.

Proof. The induction is on the number of type constructors in $\Psi$.

Theorem 11 (Decidability of typing) 1. Given $\Gamma, \Delta, R$, and $A$, it is decidable whether $\Gamma ; \Delta \vdash R \Rightarrow A$. 
2. Given $\Gamma, \Delta, N$, and $A$, it is decidable whether $\Gamma ; \Delta \vdash N \Leftarrow A$.

3. Given $\Gamma, \Delta, M$, and $S$, it is decidable whether $\Gamma ; \Delta \vdash M \Leftarrow S$.

4. Given $\Gamma, \Delta, E$, and $S$, it is decidable whether $\Gamma ; \Delta \vdash E \leftarrow S$.

5. Given $\Gamma, P$, and $K$, it is decidable whether $\Gamma \vdash P \Rightarrow K$.

6. Given $\Gamma$ and $A$, it is decidable whether $\Gamma \vdash A \Leftarrow$ type.

7. Given $\Gamma$ and $K$, it is decidable whether $\Gamma \vdash K \Leftarrow$ kind.

8. Given $\Sigma$, it is decidable whether $\vdash \Sigma$ ok.

9. Given $\Gamma$, it is decidable whether $\vdash \Gamma$ ok.

10. Given $\Gamma$ and $\Delta$, it is decidable whether $\Gamma \vdash \Delta$ ok.

11. Given $\Gamma$ and $\Psi$, it is decidable whether $\Gamma \vdash \Psi$ ok.

Proof. The proof is by structural induction on the subject of each judgment. In order to decide $\Gamma ; \Delta \vdash R \Leftarrow P$, we test whether typeof $(\Gamma ; \Delta \vdash R)$ is atomic, typeof $(\Gamma ; \Delta \vdash R) \equiv P$, and $\Gamma ; \Delta \vdash R \Rightarrow \operatorname{typeof}(\Gamma ; \Delta \vdash R)$. In order to decide $\Gamma ; \Delta \vdash R N \Rightarrow A$, we first test whether typeof $(\Gamma ; \Delta \vdash R) \equiv \Pi x: B$. $C$ for some $B$ and $C$, whether $\Gamma ; \Delta \vdash R \Rightarrow \Pi x: B . C$, whether $\Gamma ; \cdot \vdash N \Leftarrow B$, and finally whether inst_a ${ }_{B}(x . C, N) \equiv A$. In order to decide $\Gamma ; \Delta \vdash$ let $\{p\}=R$ in $E \leftarrow S$ there is an appeal to the lemma. Similar comments apply in other cases. In order to decide multiplicatives, it suffices - efficiency concerns aside - to test every possible decomposition of the linear context $\Delta$ into $\Delta_{1}, \Delta_{2}$.

It is characteristic of this approach to the type theory that such decidability theorems can be proved before any of the other meta-theory is developed, and that they do not depend in any way on the various terms involved being valid. The most important reason is that every judgment and algorithm of the theory is syntax directed.

\subsection{Composition}

A novel element of the meta-theory is the need for composition theorems for the instantiation and expansion operators. These correspond to the usual categorical axioms of left and right identity and associativity (slightly modified because we instantiate a single variable at a time rather than all free variables). In a language admitting non-canonical forms these are trivial, since the identity principle is witnessed trivially, and there is a composition law for syntactic substitution:

$$
\left[M_{1} / x_{1}\right]\left[M_{2} / x_{2}\right] M_{3} \equiv\left[\left[M_{1} / x_{1}\right] M_{2}\right]\left[M_{1} / x_{1}\right] M_{3}
$$

The main theorem of this section is a corresponding law for instantiation. We begin with a number of lemmas.

Lemma 12 (Trivial instantiation) 1. If $x \notin \mathrm{FV}(R)$, then inst_r ${ }_{A}\left(x . R, N_{0}\right) \equiv R$.

2. If $x \notin \mathrm{FV}(N)$, then inst_n $\mathrm{n}_{A}\left(x . N, N_{0}\right) \equiv N$.

3. If $x \notin \mathrm{FV}(M)$, then inst_m ${ }_{A}\left(x . M, N_{0}\right) \equiv M$.

4. If $x \notin \mathrm{FV}(E)$, then inst_e $A_{A}\left(x . E, N_{0}\right) \equiv E$.

5. If $x \notin \mathrm{FV}(P)$, then inst_p ${ }_{A}\left(x . P, N_{0}\right) \equiv P$.

6. If $x \notin \mathrm{FV}(B)$, then inst_a $A\left(x . B, N_{0}\right) \equiv B$. 
7. If $x \notin \mathrm{FV}(S)$, then inst_s $A\left(x . S, N_{0}\right) \equiv S$.

8. If $x \notin \mathrm{FV}(K)$, then inst_k ${ }_{A}\left(x . K, N_{0}\right) \equiv K$.

Proof. A straightforward induction on the first argument.

LEMMA 13

If inst_r ${ }_{A}\left(x . R, N_{0}\right)$ is defined, then head(inst_r $\left.{ }_{A}\left(x . R, N_{0}\right)\right)$ is head $(R)$.

LEMMA 14

If $x$ and $y$ are distinct, treduce ${ }_{A}(x . R) \equiv A^{\prime}$, and inst_r ${ }_{B}\left(y . R, N_{0}\right)$ is defined, then

$$
\text { treduce }_{A}\left(x \text {. inst_r }{ }_{B}\left(y \cdot R, N_{0}\right)\right) \equiv A^{\prime} .
$$

LEMMA 15

The free variables in a term resulting from instantiating $x$ with $N_{0}$ are a subset of the free variables of the original term, excepting $x$.

The free variables in a term resulting from matching $p$ with $E_{0}$ or $M_{0}$ are a subset of the free variables of the original term, excepting the variables of $p$.

Note that the following theorem holds without assuming that the terms involved are well-typed.

\section{Theorem 16 (Composition of instantiations)}

Suppose that $x$ and $y$ are distinct or $x \notin \mathrm{FV}(p)$, and $y \notin \mathrm{FV}\left(N_{0}\right)$ or $\mathrm{FV}(p) \cap \mathrm{FV}\left(N_{0}\right)$ is empty, as the case may be. For each of the following equations, if the inner instantiations are defined, then the outermost instantiations on each side are both defined and are $\alpha$ equivalent.

1. reduce $_{A_{0}}\left(x\right.$. inst_r $\left.{ }_{A}\left(y \cdot R_{1}, N_{2}\right), N_{0}\right) \equiv$ inst_n $_{A}\left(y\right.$. reduce $A_{0}\left(x \cdot R_{1}, N_{0}\right)$, inst_n $\left._{A_{0}}\left(x \cdot N_{2}, N_{0}\right)\right)$

2. inst $\_\mathrm{n}_{A_{0}}\left(x\right.$. reduce $\left.{ }_{A}\left(y \cdot R_{1}, N_{2}\right), N_{0}\right) \equiv$ reduce $_{A}\left(y\right.$. inst_r ${ }_{A_{0}}\left(x . R_{1}, N_{0}\right)$, inst_n $\left.\mathrm{n}_{A_{0}}\left(x \cdot N_{2}, N_{0}\right)\right)$

3 . inst $r_{A_{0}}\left(x\right.$. inst_r $\left.r_{A}\left(y \cdot R_{1}, N_{2}\right), N_{0}\right) \equiv$ inst_r $_{A}\left(y\right.$. inst_r $A_{A_{0}}\left(x . R_{1}, N_{0}\right)$, inst_n $\left.A_{A_{0}}\left(x \cdot N_{2}, N_{0}\right)\right)$

4. inst_n $\mathrm{n}_{A_{0}}\left(x\right.$. inst_n $\left.\mathrm{n}_{A}\left(y \cdot N_{1}, N_{2}\right), N_{0}\right) \equiv$ inst_n $_{A}\left(y\right.$. inst_n $\mathrm{n}_{A_{0}}\left(x . N_{1}, N_{0}\right)$, inst_n $\left.\mathrm{n}_{A_{0}}\left(x \cdot N_{2}, N_{0}\right)\right)$

5. inst_m $A_{A_{0}}\left(x\right.$. inst_m $\left.{ }_{A}\left(y \cdot M_{1}, N_{2}\right), N_{0}\right) \equiv$ inst_m ${ }_{A}\left(y\right.$. inst_m $_{A_{0}}\left(x \cdot M_{1}, N_{0}\right)$, inst_n $\left.{ }_{A_{0}}\left(x \cdot N_{2}, N_{0}\right)\right)$

6 . inst_e $A_{0}\left(x\right.$. inst_e $\left.e_{A}\left(y . E_{1}, N_{2}\right), N_{0}\right) \equiv$ inst_e $_{A}\left(y\right.$. inst_e $e_{A_{0}}\left(x . E_{1}, N_{0}\right)$, inst_n $\left.A_{A_{0}}\left(x . N_{2}, N_{0}\right)\right)$

7. inst_e $A_{0}\left(x\right.$. match_m $\left.\mathrm{m}_{S}\left(p . E_{1}, M_{2}\right), N_{0}\right) \equiv$ match_m ${ }_{S}\left(p\right.$. inst_e $A_{0}\left(x . E_{1}, N_{0}\right)$, inst_m $\left.{ }_{A_{0}}\left(x . M_{2}, N_{0}\right)\right)$

8. inst_e $e_{A_{0}}\left(x\right.$. match_e $\left.\mathrm{e}_{S}\left(p . E_{1}, E_{2}\right), N_{0}\right) \equiv$ match_e $\mathrm{e}_{A}\left(p\right.$. inst_e $\mathrm{e}_{A_{0}}\left(x . E_{1}, N_{0}\right)$, inst_e $\left.\mathrm{e}_{A_{0}}\left(x . E_{2}, N_{0}\right)\right)$

9. match_m $\mathrm{m}_{S_{0}}\left(p_{0}\right.$. match_e $\left.{ }_{S}\left(p . E_{1}, E_{2}\right), M_{0}\right)$

$$
\equiv \text { match_e } e_{S}\left(p \text {. match } \_\mathrm{m}_{S_{0}}\left(p_{0} . E_{1}, M_{0}\right) \text {, match_m } \mathrm{m}_{S_{0}}\left(p_{0} . E_{2}, M_{0}\right)\right)
$$

10. match_e $S_{S_{0}}\left(p_{0}\right.$. match_e $\left.{ }_{S}\left(p . E_{1}, E_{2}\right), E_{0}\right) \equiv \operatorname{match\_ } \mathrm{e}_{S}\left(p . E_{1}\right.$, match_e $\left.e_{S_{0}}\left(p_{0} . E_{2}, E_{0}\right)\right)$

(Supposing no variable bound by $p_{0}$ is free in $E_{1}$.)

11. inst_p $A_{A_{0}}\left(x\right.$. inst_p $\left.{ }_{A}\left(y . P_{1}, N_{2}\right), N_{0}\right) \equiv$ inst_p $_{A}\left(y\right.$. inst_p ${ }_{A_{0}}\left(x \cdot P_{1}, N_{0}\right)$, inst_n $\left.A_{A_{0}}\left(x \cdot N_{2}, N_{0}\right)\right)$

12. inst_a $A_{0}\left(x\right.$. inst_a $\left.A\left(y . A_{1}, N_{2}\right), N_{0}\right) \equiv$ inst_a $_{A}\left(y\right.$. inst_a $A_{0}\left(x . A_{1}, N_{0}\right)$, inst_n $\left.A_{A_{0}}\left(x . N_{2}, N_{0}\right)\right)$

13. inst $\_s_{A_{0}}\left(x\right.$. inst_s $\left.s_{A}\left(y . S_{1}, N_{2}\right), N_{0}\right) \equiv$ inst_s $s_{A}\left(y\right.$. inst_s $s_{A_{0}}\left(x . S_{1}, N_{0}\right)$, inst_n $\left.\mathrm{n}_{A_{0}}\left(x . N_{2}, N_{0}\right)\right)$

14. inst_k $\mathrm{k}_{A_{0}}\left(x\right.$. inst_k $\left.{ }_{A}\left(y . K_{1}, N_{2}\right), N_{0}\right) \equiv$ inst_k $_{A}\left(y\right.$. inst_k ${ }_{A_{0}}\left(x . K_{1}, N_{0}\right)$, inst_n $\left.{ }_{A_{0}}\left(x . N_{2}, N_{0}\right)\right)$

Proof. The proof has many cases but is straightforward. 


\subsection{On equality}

The following theorems show that the framework's equality is an equivalence relation.

Theorem 17 (REFleXivity of EQUAlity) 1. Given $R$, we have $R=R$.

2. Given $N$, we have $N=N$.

3. Given $M$, we have $M=M$.

4. Given $E$, we have $E={ }_{\mathrm{c}} E$.

5. Given $E$, we have $E=E$.

6. Given $P$, we have $P=P$.

Proof. The proof is by structural induction on the subject of the judgment.

LEMMA 18

If $E_{1}={ }_{\mathrm{c}}$ (let $\{p\}=R_{2}$ in $E_{2}^{\prime}$ ), then $E_{1} \equiv \epsilon\left[\right.$ let $\{p\}=R_{1}$ in $\left.E_{1}^{\prime}\right]$ for some $R_{1}$, $\epsilon$ and $E_{1}^{\prime}$ such that $R_{1}=R_{2}$ and $\epsilon\left[E_{1}^{\prime}\right]={ }_{\mathrm{c}} E_{2}^{\prime}$. Furthermore, no variable free in $R_{1}$ is bound in $\epsilon$.

Proof. The proof is by structural induction on the derivation of the assumption.

Theorem 19 (Symmetry of Equality) 1. If $R_{1}=R_{2}$, then $R_{2}=R_{1}$.

2. If $N_{1}=N_{2}$, then $N_{2}=N_{1}$.

3. If $M_{1}=M_{2}$, then $M_{2}=M_{1}$.

4. If $E_{1}={ }_{\mathrm{c}} E_{2}$, then $E_{2}={ }_{\mathrm{c}} E_{1}$.

5. If $E_{1}=E_{2}$, then $E_{2}=E_{1}$.

6. If $P_{1}=P_{2}$, then $P_{2}=P_{1}$.

Proof. The proof is by structural induction on the second subject of the assumed equality. In the case $E_{1}={ }_{\mathrm{c}}$ (let $\{p\}=R_{2}$ in $E_{2}^{\prime}$ ) we appeal to the lemma. Then $E_{2}^{\prime}=_{\mathrm{c}} \epsilon\left[E_{1}^{\prime}\right]$ and $R_{2}=R_{1}$ by the induction hypothesis. Then (let $\{p\}=R_{2}$ in $\left.E_{2}^{\prime}\right)=_{\mathrm{c}} \epsilon\left[\right.$ let $\{p\}=R_{1}$ in $\left.E_{1}^{\prime}\right] \equiv$ $E_{1}$ by an inference rule.

In order to prove the transitivity of concurrent equality, we first need to consider a few trivial properties of concurrent contexts (somewhat tedious to prove syntactically).

LEMma 20

If $\epsilon\left[E_{1}\right] \equiv \epsilon\left[E_{2}\right]$, then $E_{1} \equiv E_{2}$.

Proof. The result follows by induction on $\epsilon$.

LEMma 21

If $\epsilon_{1}[E] \equiv \epsilon_{2}[E]$, then $\epsilon_{1} \equiv \epsilon_{2}$.

Proof. The result follows by induction on $\epsilon_{1}$.

Lemma 22

Suppose that no variable free in $R$ is bound in $\epsilon_{2}$. If $\epsilon_{1}\left[E_{1}\right] \equiv \epsilon_{2}\left[\right.$ let $\{p\}=R$ in $\left.E_{2}\right]$, then either

1. $\epsilon_{1} \equiv \epsilon_{2}\left[\right.$ let $\{p\}=R$ in $\left.\epsilon^{\prime}\right]$ for some $\epsilon^{\prime}$, or 
2. $E_{1} \equiv \epsilon^{\prime}\left[\right.$ let $\{p\}=R$ in $\left.E_{2}\right]$ for some $\epsilon^{\prime}$.

Proof. The result follows by induction on $\epsilon_{1}$.

Now we prove a general inversion principle for concurrent equality.

LEMMA 23 (INVERSION PRINCIPLE FOR CONCURRENT EQUALITY)

Suppose that no variable free in $R_{1}$ is bound by $\epsilon_{1}$. If $\epsilon_{1}\left[\right.$ let $\{p\}=R_{1}$ in $\left.E_{1}^{\prime}\right]={ }_{\mathrm{c}} E_{2}$, then $E_{2} \equiv \epsilon_{2}\left[\right.$ let $\{p\}=R_{2}$ in $\left.E_{2}^{\prime}\right]$ for some $\epsilon_{2}, R_{2}$, and $E_{2}^{\prime}$ such that $R_{1}=R_{2}$ and $\epsilon_{1}\left[E_{1}^{\prime}\right]=_{\mathrm{c}} \epsilon_{2}\left[E_{2}^{\prime}\right]$. Furthermore, no variable free in $R_{2}$ is bound by $\epsilon_{2}$.

Proof. The result follows by induction on $\epsilon_{1}$.

Now we can prove the transitivity theorem itself.

Theorem 24 (Transitivity of EQUALity) 1. If $R_{1}=R_{2}$ and $R_{2}=R_{3}$, then $R_{1}=R_{3}$.

2. If $N_{1}=N_{2}$ and $N_{2}=N_{3}$, then $N_{1}=N_{3}$.

3. If $M_{1}=M_{2}$ and $M_{2}=M_{3}$, then $M_{1}=M_{3}$.

4. If $E_{1}={ }_{\mathrm{c}} E_{2}$ and $E_{2}={ }_{\mathrm{c}} E_{3}$, then $E_{1}={ }_{\mathrm{c}} E_{3}$.

5. If $E_{1}=E_{2}$ and $E_{2}=E_{3}$, then $E_{1}=E_{3}$.

6. If $P_{1}=P_{2}$ and $P_{2}=P_{3}$, then $P_{1}=P_{3}$.

Proof. The proof is by structural induction on the derivation of the first assumption. For the part involving concurrent equality we appeal to the inversion principle.

It is the restriction to canonical forms inherent in the syntax of CLF that makes it possible to define equality and prove such a result without any reference to the typing judgments.

Now we go on to show that all of the primitive operators and judgments of the theory factor through the equivalence relation on well-typed terms induced by the equality judgment. (Recall that we do not ascribe any particular meaning to the equality judgment unless the terms involved are well-typed.) This licenses us to think of them as being defined on the equivalence classes.

First, however, we introduce a stronger version of concurrent equality, needed to stage the proofs. Strong concurrent equality only allows rearranging let bindings at the top level structure of an expression, rather than deep within it.

DEFinition 16 (STRong CONCURRENT EQUALity)

$E_{1}={ }_{\mathrm{S}} E_{2}$

[Strong concurrent equality]

$$
\frac{E_{1}={ }_{\mathrm{s}} \epsilon\left[E_{2}\right]}{M={ }_{\mathrm{s}} M} \quad \frac{{ }}{\left(\text { let }\{p\}=R \text { in } E_{1}\right)={ }_{\mathrm{s}} \epsilon\left[\text { let }\{p\}=R \text { in } E_{2}\right]} *
$$

Again the rule marked $\left({ }^{*}\right)$ is subject to the side condition that no variable bound by $p$ be free in the conclusion or bound by the context $\epsilon$, and that no variable free in $R$ be bound by the context $\epsilon$. We have that strong concurrent equality is reflexive, symmetric, and transitive, by essentially the same arguments as for the original concurrent equality.

THEOREM 25

Strong concurrent equality is an equivalence relation. 
Strong concurrent equality, as the name suggests, is a special case of concurrent equality. This follows from the reflexivity of framework equality.

THEOREM 26

If $E_{1}={ }_{\mathrm{s}} E_{2}$, then $E_{1}={ }_{\mathrm{c}} E_{2}$.

We also have the following lemmas.

LEMMA 27

Given $S, p, E, \epsilon$, and $E_{0}$, we have match_e $\mathrm{e}_{S}\left(p . E, \epsilon\left[E_{0}\right]\right) \equiv \epsilon\left[\right.$ match_e $\left._{S}\left(p . E, E_{0}\right)\right]$, supposing one or the other side is defined.

Proof. The result follows by induction on $\epsilon$.

LEMMA 28

Suppose that $p$ and $p^{\prime}$ bind disjoint variables, and no variable free in $R^{\prime}$ is bound by $p$, and no variable free in $E_{0}$ or $M_{0}$ is bound by $p^{\prime}$. Then the following equations hold, assuming one or the other side is defined.

1. $\operatorname{match} \_\mathrm{m}_{S}\left(p\right.$. let $\left\{p^{\prime}\right\}=R^{\prime}$ in $\left.E, M_{0}\right) \equiv\left(\right.$ let $\left\{p^{\prime}\right\}=R^{\prime}$ in match_m $\left.{ }_{S}\left(p . E, M_{0}\right)\right)$

2. match_e $\mathrm{e}_{S}\left(p\right.$. let $\left\{p^{\prime}\right\}=R^{\prime}$ in $\left.E, E_{0}\right)={ }_{\mathrm{s}}\left(\right.$ let $\left\{p^{\prime}\right\}=R^{\prime}$ in match_e ${ }_{S}\left(p\right.$. $\left.\left.E, E_{0}\right)\right)$

Proof. The first part follows by induction on $S$, and then the second part follows by induction on $E_{0}$.

LEMma 29

Suppose that $p_{1}$ and $p_{2}$ bind disjoint variables, and that no variable free in $E_{1}$ is bound by $p_{2}$, and no variable free in $E_{2}$ is bound by $p_{1}$. Then

match_e $S_{S_{1}}\left(p_{1}\right.$. match_e $\left.S_{S_{2}}\left(p_{2} . E, E_{2}\right), E_{1}\right)={ }_{\mathrm{s}}$ match_e $S_{S_{2}}\left(p_{2}\right.$. match_e $\left.{ }_{S_{1}}\left(p_{1} . E, E_{1}\right), E_{2}\right)$

as long as one or the other side is defined.

Proof. The proof is by induction on $E_{1}$. In the case $E_{1} \equiv M_{1}$ we appeal to the composition law for instantiation. Otherwise we appeal to the preceding lemma.

The utility of strong concurrent equality is that the following theorem can be proved immediately.

THEOREM 30

In each of the following cases, the resulting equality holds assuming that one side or the other is defined.

1. If no variable free in $R$ is bound by $\epsilon$, and $\epsilon$ and $p$ bind disjoint sets of variables, then inst_e $_{A}\left(x\right.$. let $\{p\}=R$ in $\left.\epsilon[E], N_{0}\right)={ }_{\text {s inst_e }}\left(x\right.$. $\epsilon[$ let $\{p\}=R$ in $\left.E], N_{0}\right)$.

2. If $E={ }_{\mathrm{S}} E^{\prime}$, then inst_e ${ }_{A}\left(x . E, N_{0}\right)={ }_{\mathrm{s}}$ inst_e $_{A}\left(x . E^{\prime}, N_{0}\right)$.

3. If $E={ }_{\mathrm{s}} E^{\prime}$, then match_m $\mathrm{m}_{S}\left(p . E, M_{0}\right)={ }_{\mathrm{s}} \operatorname{match} \_\mathrm{m}_{S}\left(p . E^{\prime}, M_{0}\right)$.

4. If $E={ }_{\mathrm{s}} E^{\prime}$, then match_e $\mathrm{e}_{S}\left(p . E, E_{0}\right)={ }_{\mathrm{s}} \operatorname{match} \mathrm{e}_{S}\left(p . E^{\prime}, E_{0}\right)$. 
Proof. The proof is by an outer induction on the type subscript. The first part also uses an inner induction on $\epsilon$, the second part uses an inner induction on the derivation of $E={ }_{\mathrm{s}} E^{\prime}$, and the last part uses an inner induction on $E_{0}$.

We are now in a position to prove the "functionality" of instantiation with respect to equivalence classes modulo the framework's equality. We extend equality to types and kinds by the natural congruence rules.

Theorem 31 (FUnCTIONALITY FOR Instantiation)

In each of the following cases, the resulting equality holds assuming that one side or the other is defined.

1. If $R=R^{\prime}$ and $N_{0}=N_{0}^{\prime}$, then reduce $_{A}\left(x . R, N_{0}\right)=$ reduce $_{A}\left(x . R^{\prime}, N_{0}^{\prime}\right)$.

2. If $R=R^{\prime}$ and $N_{0}=N_{0}^{\prime}$, then inst_r ${ }_{A}\left(x . R, N_{0}\right)=$ inst_r $_{A}\left(x . R^{\prime}, N_{0}^{\prime}\right)$.

3. If $N=N^{\prime}$ and $N_{0}=N_{0}^{\prime}$, then inst_n $\mathrm{n}_{A}\left(x . N, N_{0}\right)=$ inst_n $_{A}\left(x . N^{\prime}, N_{0}^{\prime}\right)$.

4. If $M=M^{\prime}$ and $N_{0}=N_{0}^{\prime}$, then inst_m ${ }_{A}\left(x . M, N_{0}\right)=$ inst_m $_{A}\left(x . M^{\prime}, N_{0}^{\prime}\right)$.

5. If $E=E^{\prime}$ and $N_{0}=N_{0}^{\prime}$, then inst_e ${ }_{A}\left(x . E, N_{0}\right)=$ inst_e $_{A}\left(x . E^{\prime}, N_{0}^{\prime}\right)$.

6. If $E=E^{\prime}$ and $M_{0}=M_{0}^{\prime}$, then match_m $\mathrm{m}_{S}\left(p . E, M_{0}\right)=$ match_m $\mathrm{m}_{S}\left(p . E^{\prime}, M_{0}^{\prime}\right)$.

7. If $E=E^{\prime}$ and $E_{0}=E_{0}^{\prime}$, then match_e $\mathrm{e}_{S}\left(p . E, E_{0}\right)=$ match_e ${ }_{S}\left(p \cdot E^{\prime}, E_{0}^{\prime}\right)$.

8. If $P=P^{\prime}$ and $N_{0}=N_{0}^{\prime}$, then inst_p ${ }_{A}\left(x . P, N_{0}\right)=$ inst_p ${ }_{A}\left(x . P^{\prime}, N_{0}^{\prime}\right)$.

9. If $B=B^{\prime}$ and $N_{0}=N_{0}^{\prime}$, then inst_a ${ }_{A}\left(x . B, N_{0}\right)=$ inst_a $_{A}\left(x . B^{\prime}, N_{0}^{\prime}\right)$.

10. If $S=S^{\prime}$ and $N_{0}=N_{0}^{\prime}$, then inst_s ${ }_{A}\left(x . S, N_{0}\right)=$ inst_s $_{A}\left(x . S^{\prime}, N_{0}^{\prime}\right)$.

11. If $K=K^{\prime}$ and $N_{0}=N_{0}^{\prime}$, then inst_k ${ }_{A}\left(x . K, N_{0}\right)=$ inst_k $_{A}\left(x . K^{\prime}, N_{0}^{\prime}\right)$.

Proof. The proof is by an outer induction on the type subscript and an inner simultaneous induction on the derivations of the two assumed equalities.

In most cases the result follows immediately by congruence rules. The critical parts are the ones given by induction over expressions. For match_e ${ }_{S}\left(p . E, E_{0}\right)={ }_{\mathrm{c}}$ match_e ${ }_{S}\left(p . E^{\prime}, E_{0}^{\prime}\right)$ we appeal to Lemma 27.

For inst_e ${ }_{A}\left(x . E, N_{0}\right)={ }_{\mathrm{c}}$ inst_e ${ }_{A}\left(x . E^{\prime}, N_{0}^{\prime}\right)$ we reason as follows. If $E \equiv M$, the result is immediate by the induction hypothesis. Otherwise $E \equiv$ (let $\{p\}=R$ in $E_{1}$ ), $E^{\prime} \equiv$ $\epsilon\left[\right.$ let $\{p\}=R^{\prime}$ in $\left.E_{1}^{\prime}\right], R=R^{\prime}$, and $E_{1}=_{\mathrm{c}} \epsilon\left[E_{1}^{\prime}\right]$. Then

$$
\text { inst_e }{ }_{A}\left(x . E_{1}, N_{0}\right)={ }_{\mathrm{c}} \text { inst_e }{ }_{A}\left(x . \epsilon\left[E_{1}^{\prime}\right], N_{0}^{\prime}\right)
$$

by the induction hypothesis. It follows that

$$
\text { inst_e }_{A}\left(x \text {. let }\{p\}=R \text { in } E_{1}, N_{0}\right)={ }_{\mathrm{c}} \text { inst_e }{ }_{A}\left(x \text {. let }\{p\}=R^{\prime} \text { in } \epsilon\left[E_{1}^{\prime}\right], N_{0}^{\prime}\right)
$$

by another appeal to the induction hypothesis. But by the preceding theorem,

$$
\text { inst_e }{ }_{A}\left(x \text {. let }\{p\}=R^{\prime} \text { in } \epsilon\left[E_{1}^{\prime}\right], N_{0}^{\prime}\right)={ }_{\mathrm{s}} \text { inst_e }{ }_{A}\left(x . \epsilon\left[\text { let }\{p\}=R^{\prime} \text { in } E_{1}^{\prime}\right], N_{0}^{\prime}\right) \text {. }
$$

The result then follows by transitivity.

A similar result holds for the expansion operator. 
THEOREM 32 (FUnCTIONALITY FOR EXPANSION)

If $R=R^{\prime}$, then expand $_{A}(R)=\operatorname{expand}_{A}\left(R^{\prime}\right)$.

Proof. The proof is by induction on $A$.

The functionality of instantiation immediately leads to a similar result for the typeof and kindof operators of Lemma 9. We extend equality to contexts and signatures in the obvious way.

LEMMA 33

If $\Sigma=\Sigma^{\prime}, \Gamma=\Gamma^{\prime}$, and $\Delta=\Delta^{\prime}$, then typeof $\left(\Gamma ; \Delta \vdash_{\Sigma} R\right)=\operatorname{typeof}\left(\Gamma^{\prime} ; \Delta^{\prime} \vdash_{\Sigma^{\prime}} R\right)$ and kindof $\left(\Gamma \vdash_{\Sigma} P\right)=\operatorname{kindof}\left(\Gamma^{\prime} \vdash_{\Sigma^{\prime}} P\right)$, supposing one or the other side is defined.

It is characteristic of the syntax-directed approach that the following theorem holds even when the contexts, etc. are not valid.

THEOREM 34 (TYPE CONVERSION)

Suppose that $\Sigma=\Sigma^{\prime}, \Gamma=\Gamma^{\prime}, \Delta=\Delta^{\prime}, A=A^{\prime}$, and $S=S^{\prime}$.

1. $\Gamma ; \Delta \vdash_{\Sigma} R \Rightarrow$ typeof $\left(\Gamma ; \Delta \vdash_{\Sigma} R\right)$ iff $\Gamma^{\prime} ; \Delta^{\prime} \vdash_{\Sigma^{\prime}} R \Rightarrow$ typeof $\left(\Gamma^{\prime} ; \Delta^{\prime} \vdash_{\Sigma^{\prime}} R\right)$.

2. $\Gamma ; \Delta \vdash_{\Sigma} N \Leftarrow A$ iff $\Gamma^{\prime} ; \Delta^{\prime} \vdash_{\Sigma^{\prime}} N \Leftarrow A^{\prime}$.

3. $\Gamma ; \Delta \vdash_{\Sigma} M \Leftarrow S$ iff $\Gamma^{\prime} ; \Delta^{\prime} \vdash_{\Sigma^{\prime}} M \Leftarrow S^{\prime}$.

4. $\Gamma ; \Delta \vdash_{\Sigma} E \leftarrow S$ iff $\Gamma^{\prime} ; \Delta^{\prime} \vdash_{\Sigma^{\prime}} E \leftarrow S^{\prime}$.

5. $\Gamma \vdash_{\Sigma} P \Rightarrow \operatorname{kindof}\left(\Gamma \vdash_{\Sigma} P\right)$ iff $\Gamma^{\prime} \vdash_{\Sigma^{\prime}} P \Rightarrow \operatorname{kindof}\left(\Gamma^{\prime} \vdash_{\Sigma^{\prime}} P\right)$.

6. $\Gamma \vdash_{\Sigma} A \Leftarrow$ type iff $\Gamma^{\prime} \vdash_{\Sigma^{\prime}} A \Leftarrow$ type.

7. $\Gamma \vdash_{\Sigma} S \Leftarrow$ type iff $\Gamma^{\prime} \vdash_{\Sigma^{\prime}} S \Leftarrow$ type.

8. $\Gamma \vdash_{\Sigma} K \Leftarrow$ kind iff $\Gamma^{\prime} \vdash_{\Sigma^{\prime}} K \Leftarrow$ kind.

Proof. The theorem is proved by induction over the assumed typing derivation, using Lemma 9 .

\subsection{On typing}

Now we can go on to prove the substitution and identity principles for CLF, and that they are witnessed by the instantiation and expansion operators. The instantiation operators are extended to contexts in the obvious way. Again, the theorem holds even when the contexts are not valid.

Theorem 35 (Substitution PRINCIPLES)

If $\Gamma_{\mathrm{L}} ; \cdot \vdash N_{0} \Leftarrow A$ is derivable, $\Gamma_{\mathrm{L}}, x: A, \Gamma_{\mathrm{R}} ; \Delta \vdash N \Leftarrow C$ is derivable, inst_a ${ }_{A}\left(x . \Gamma_{\mathrm{R}}, N_{0}\right) \equiv$ $\Gamma_{\mathrm{R}}^{\prime}$, inst_a $a_{A}\left(x . \Delta, N_{0}\right) \equiv \Delta^{\prime}$, and inst_a ${ }_{A}\left(x . C, N_{0}\right) \equiv C^{\prime}$, the following hold:

1. The instantiation inst_ $\mathrm{n}_{A}\left(x . N, N_{0}\right)$ is defined.

2. The judgment $\Gamma_{\mathrm{L}}, \Gamma_{\mathrm{R}}^{\prime} ; \Delta^{\prime} \vdash$ inst_n $\mathrm{n}_{A}\left(x . N, N_{0}\right) \Leftarrow C^{\prime}$ is derivable.

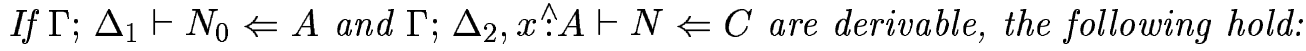

1. The instantiation inst_ $\mathrm{n}_{A}\left(x . N, N_{0}\right)$ is defined. 
2. The judgment $\Gamma ; \Delta_{1}, \Delta_{2} \vdash$ inst_n $\mathrm{n}_{A}\left(x . N, N_{0}\right) \Leftarrow C$ is derivable.

This result is mutually dependent on many other substitution theorems for the other syntactic categories. Space considerations preclude the incorporation of the proof here. It is notable that although the theorem as a whole does not assume the contexts are valid, in each case "just enough" information about the validity of various terms is available in order to make the induction go through.

Note that the presence of the type subscript to the instantiation operators is redundant if the objects involved are known to be well typed, since the types generated as reduce decomposes a well-typed object will always be what they are required to be. An optimized type checker can take advantage of this by staging the process of type checking so that it is an invariant that whenever an instantiation is applied the objects involved are known to be well typed. The fact that this is possible is evident upon examination of the flow of information through the typing rules.

The following theorem can be proved by a simple structural induction.

LEMMA 36 (EXPANSION)

If $\Gamma ; \Delta \vdash R \Rightarrow A$ then $\Gamma ; \Delta \vdash \operatorname{expand}_{A}(R) \Leftarrow A$.

As an immediate corollary, we have the following identity property.

THEOREM 37 (IDENTITY PRINCIPLES)

Any instance of $\Gamma, x: A ; \cdot \vdash \operatorname{expand}_{A}(x) \Leftarrow A$ or $\Gamma ; x \wedge A \vdash \operatorname{expand}_{A}(x) \Leftarrow A$ is derivable.

It is worth recalling that the substitution and identity principles are are needed to ensure that the type theory makes sense, since the syntatic restrictions inherent in CLF make it impossible to generate proofs of $A \rightarrow A$ or to compose proofs of $A \rightarrow B$ and $B \rightarrow C$ in any other way.

\subsection{Related work}

With one exception [Fel91], prior presentations of LF and LLF have been based on a syntax in which not every term is canonical. A difficulty is that equality cannot then be axiomatized in a manifestly decidable, syntax-directed way. In their original presentation of LF, Harper et al. define equality in terms of $\beta$-convertibility, and do not address $\eta$-conversions [HHP93]. Strong normalization ensures that this notion of equality is decidable. However, the $\eta$ conversions pose a special difficulty because of the lack of confluence for $\beta \eta$-reduction in the case of non-well-typed terms. Since LF typing is dependent on equality, the attempt to define an equality based on $\beta \eta$-reduction leads to a Catch-22.

Coquand [Coq91] tests $\beta \eta$-convertibility in LF using untyped $\beta$-reduction and extensionality, which is applied when comparing a $\lambda$-abstraction to a non-abstraction. However, this method fails when a unit type is present - as in LLF - because it may be necessary to apply extensionality even when neither of the terms being compared is a manifest unit introduction.

Cervesato's presentation of LLF avoids the $\eta$-conversion problem by restricting the syntax to $\eta$-long terms [Cer96]. The equality of the framework is still defined in terms of $\beta$-reduction. This is possible because the $\beta$-reducts of $\eta$-long terms are $\eta$-long.

Goguen proposes an elegant theory based on a typed notion of reduction [Gog94, Gog99]. An operational semantics based on this typed reduction is then shown to be decidable, and 
equivalent to a (not manifestly decidable) axiomatization of equality from first principles such as extensionality. However, being based on $\eta$-reduction, this approach does not decide equality by an analysis of canonical forms, which are $\eta$-long. This conflicts with the LF representation methodology, which emphasizes the primacy of canonical forms in constructing representations and proving their adequacy.

Ghani [Gha97] uses a typed rewriting relation similar to Goguen's operational semantics but with $\eta$-expansion rather than $\eta$-reduction. This leads to a more pleasant theory, especially given that normal forms with respect to Ghani's rewrite rule are canonical. Harper and Pfenning [HP00] also adopt an approach similar to Goguen's, in that equality is defined axiomatically and shown to be equivalent to a decision procedure. Their method improves on Goguen's in that the decision procedure is based on transforming a pair of terms simultaneously into canonical form. It offers the further advantage that the transformation into canonical form is incremental and can be aborted as soon as it is evident that the canonical forms of the two terms being compared will not be the same, an important concern for efficient implementation.

Felty has described a canonical $L F$ in which only canonical forms are well-typed [Fel91]. This offers a number of advantages over other approaches: equality itself need not be axiomatized at all, because terms are equal just when they are identical (up to $\alpha$-equivalence). And the representation methodology has an attractive simplicity: one establishes a compositional bijection between object-language terms and LF objects of a given type. One need not restrict the range of the bijection to "canonical LF terms" because every term in canonical LF is "canonical." However, Felty's development falls back on untyped $\beta$-reduction in order to define the typing judgment on canonical terms, so a syntax of non-canonical terms ends up being reintroduced after all, and strong normalization and confluence for the non-canonical forms must be proved. Thus, the canonical language cannot be considered foundational.

Relative to these prior developments, a contribution of this work is to elaborate a foundation for LF and LLF that preserves the attractive features of Felty's canonical LF, while eliminating entirely its dependence on non-canonical terms and $\beta$-reduction, in favor of a instantiation operator taking canonical terms into canonical terms. The key observation is that the instantiation operator can be defined in a manifestly terminating, syntax-directed way, even over ill-typed terms. This essentially eliminates the mutual dependence of typing and equality - since extensionality principles depend on typing - that is inherent in Goguen's or Harper and Pfenning's work. However, it is important to stress that this approach provides only a foundation. An efficient implementation would need to reintroduce defined constants and explicit substitutions, each of which would make the equality on the LF fragment non-trivial again. But in contrast to previous approaches, the framework is defined without any reference to such "non-canonical" forms: it can scale up to include them, but its foundation is independent of them.

\section{Conclusion}

We have seen that representations of concurrent systems can be succinctly and straightforwardly constructed using a logical framework with a notion of equality that models concurrency. We have shown that the framework is decidable and investigated its metatheory. We hope that the result of this work will be a concrete language in which it is as unnecessary to specify or think about the low-level mechanics of the representation of con- 
current computations as it would be to specify or think about matters of $\alpha$-conversion and capture-avoiding substitution in LF. Furthermore, it is to be hoped that the direct, modular and scalable account of the type theory proposed here will provide a solid foundation for future explorations within the LF family of frameworks, of ideas such as proof irrelevance and ordered hypothetical judgments.

\section{A Syntax and judgments of CLF}

\section{A.1 Syntax}

DEFinition 17 (TyPE CONSTRUCTORS)

$$
\begin{aligned}
A, B, C & ::=A \multimap B|\Pi x: A . B| A \& B|\top|\{S\} \mid P & & \text { Asynchronous types } \\
P & ::=a \mid P N & & \text { Atomic type constructo } \\
S & ::=S_{1} \otimes S_{2}|1| \exists x: A . S \mid A & & \text { Synchronous types }
\end{aligned}
$$

DEFINITION 18 (KINDS)

$$
K, L::=\text { type } \mid \Pi x: A . K \quad \text { Kinds }
$$

DeFinition 19 (OBJECts)

$$
\begin{array}{rlrl}
N:: & =\hat{\lambda} x . N|\lambda x . N|\left\langle N_{1}, N_{2}\right\rangle|\langle\rangle|\{E\} \mid R & & \text { Normal objects } \\
R: & :=c|x| R^{\wedge} N|R N| \pi_{1} R \mid \pi_{2} R & & \text { Atomic objects } \\
E::=\text { let }\{p\}=R \text { in } E \mid M & & \text { Expressions } \\
M::=M_{1} \otimes M_{2}|1|[N, M] \mid N & & \text { Monadic objects } \\
p::=p_{1} \otimes p_{2}|1|[x, p] \mid x & & \text { Patterns }
\end{array}
$$

\section{A.2 Equality}

Definition 20 (CONCURRENT CONTEXts)

$$
\epsilon::={ }_{-} \mid \text {let }\{p\}=R \text { in } \epsilon \quad \text { Concurrent contexts }
$$

DEFINITION 21 (EQUALITY)

$E_{1}={ }_{\mathrm{c}} E_{2}$

[Concurrent equality]

$$
\frac{M_{1}=M_{2}}{M_{1}={ }_{\mathrm{c}} M_{2}} \quad \frac{R_{1}=R_{2} \quad E_{1}={ }_{\mathrm{c}} \epsilon\left[E_{2}\right]}{\left(\text { let }\{p\}=R_{1} \text { in } E_{1}\right)={ }_{\mathrm{c}} \epsilon\left[\text { let }\{p\}=R_{2} \text { in } E_{2}\right]} *
$$

$E_{1}=E_{2}$

[Expression equality]

$$
\frac{E_{1}={ }_{\mathrm{c}} E_{2}}{E_{1}=E_{2}}
$$


$N_{1}=N_{2} \quad R_{1}=R_{2} \quad M_{1}=M_{2} \quad P_{1}=P_{2}$

[Other equalities]

(All congruences.)

The rule marked $\left(^{*}\right)$ is subject to the side condition that no variable bound by $p$ be free in the conclusion or bound by the context $\epsilon$, and that no variable free in $R_{2}$ be bound by the context $\epsilon$.

\section{A.3 Instantiation}

Definition 22 (Instantiation)

$$
\begin{aligned}
& \text { treduce }_{A}(x . R) \equiv B \\
& \text { [Type reduction] } \\
& \text { treduce }_{A}(x . x) \equiv A \\
& \text { treduce }_{A}(x . R N) \equiv C \quad \text { if treduce } A(x . R) \equiv \Pi y: B . C \\
& \text { treduce }_{A}\left(x . R^{\wedge} N\right) \equiv C \quad \text { if treduce } A(x . R) \equiv B \multimap C \\
& \text { treduce }_{A}\left(x . \pi_{1} R\right) \equiv B_{1} \quad \text { if treduce }{ }_{A}(x . R) \equiv B_{1} \& B_{2} \\
& \text { treduce }_{A}\left(x . \pi_{2} R\right) \equiv B_{2} \quad \text { if treduce } A(x . R) \equiv B_{1} \& B_{2} \\
& \text { reduce }_{A}\left(x . R, N_{0}\right) \equiv N^{\prime} \\
& \text { [Reduction] } \\
& \text { reduce }_{A}\left(x . x, N_{0}\right) \equiv N_{0} \\
& \text { reduce }_{A}\left(x . R N, N_{0}\right) \equiv \text { inst_n }_{B}\left(y . N^{\prime}, \text { inst_n }_{A}\left(x . N, N_{0}\right)\right) \\
& \text { if treduce }{ }_{A}(x . R) \equiv \Pi y: B . C \text { and reduce }{ }_{A}\left(x . R, N_{0}\right) \equiv \lambda y . N^{\prime} \\
& \text { reduce }_{A}\left(x . R^{\wedge} N, N_{0}\right) \equiv \text { inst_n }_{B}\left(y . N^{\prime} \text {, inst_n }{ }_{A}\left(x . N, N_{0}\right)\right) \\
& \text { if treduce } \text { tr }_{A}(x) \equiv B \multimap C \text { and reduce }{ }_{A}\left(x . R, N_{0}\right) \equiv \hat{\lambda} y \cdot N^{\prime} \\
& \text { reduce }_{A}\left(x . \pi_{1} R, N_{0}\right) \equiv N_{1}^{\prime} \quad \text { if reduce } A\left(x . R, N_{0}\right) \equiv\left\langle N_{1}^{\prime}, N_{2}^{\prime}\right\rangle \\
& \text { reduce }_{A}\left(x . \pi_{2} R, N_{0}\right) \equiv N_{2}^{\prime} \quad \text { if reduce }{ }_{A}\left(x . R, N_{0}\right) \equiv\left\langle N_{1}^{\prime}, N_{2}^{\prime}\right\rangle \\
& \text { inst_r }{ }_{A}\left(x . R, N_{0}\right) \equiv R^{\prime} \\
& \text { inst_r }_{A}\left(x . R N, N_{0}\right) \equiv\left(\text { inst_r }_{A}\left(x . R, N_{0}\right)\right)\left(\text { inst_n }_{A}\left(x . N, N_{0}\right)\right) \\
& \text { inst_r }_{A}\left(x . R^{\wedge} N, N_{0}\right) \equiv\left(\text { inst_r }_{A}\left(x . R, N_{0}\right)\right)^{\wedge}\left(\text { inst_r }_{A}\left(x . N, N_{0}\right)\right) \\
& \text { inst_r }_{A}\left(x . \pi_{1} R, N_{0}\right) \equiv \pi_{1}\left(\text { inst_r }_{A}\left(x . R, N_{0}\right)\right) \\
& \text { inst_r }_{A}\left(x . \pi_{2} R, N_{0}\right) \equiv \pi_{2}\left(\text { inst_r }_{A}\left(x . R, N_{0}\right)\right)
\end{aligned}
$$

inst_n ${ }_{A}\left(x . N, N_{0}\right) \equiv N^{\prime}$

[Normal object instantiation]

$$
\begin{aligned}
& \text { inst_n } \mathrm{n}_{A}\left(x . \lambda y \cdot N, N_{0}\right) \equiv \lambda y \cdot \text { inst_n }_{A}\left(x . N, N_{0}\right) \quad \text { if } y \notin \mathrm{FV}\left(N_{0}\right) \\
& \text { inst_n }_{A}\left(x . \hat{\lambda} y . N, N_{0}\right) \equiv \hat{\lambda} y \text {. inst_n } \mathrm{n}_{A}\left(x . N, N_{0}\right) \quad \text { if } y \notin \mathrm{FV}\left(N_{0}\right) \\
& \text { inst_n }_{A}\left(x .\left\langle N_{1}, N_{2}\right\rangle, N_{0}\right) \equiv\left\langle\text { inst_n }_{A}\left(x . N_{1}, N_{0}\right) \text {, inst_n }{ }_{A}\left(x . N_{2}, N_{0}\right)\right\rangle \\
& \text { inst_n } \mathrm{n}_{A}\left(x .\langle\rangle, N_{0}\right) \equiv\langle\rangle \\
& \text { inst_n }_{A}\left(x .\{E\}, N_{0}\right) \equiv\left\{\text { inst_e }_{A}\left(x . E, N_{0}\right)\right\} \\
& \text { inst_n }_{A}\left(x . R, N_{0}\right) \equiv \text { inst_r }_{A}\left(x . R, N_{0}\right) \text { if head }(R) \text { is not } x \\
& \text { inst_n }_{A}\left(x . R, N_{0}\right) \equiv \operatorname{reduce}_{A}\left(x . R, N_{0}\right) \quad \text { if } \operatorname{treduce}_{A}(x . R) \equiv P
\end{aligned}
$$




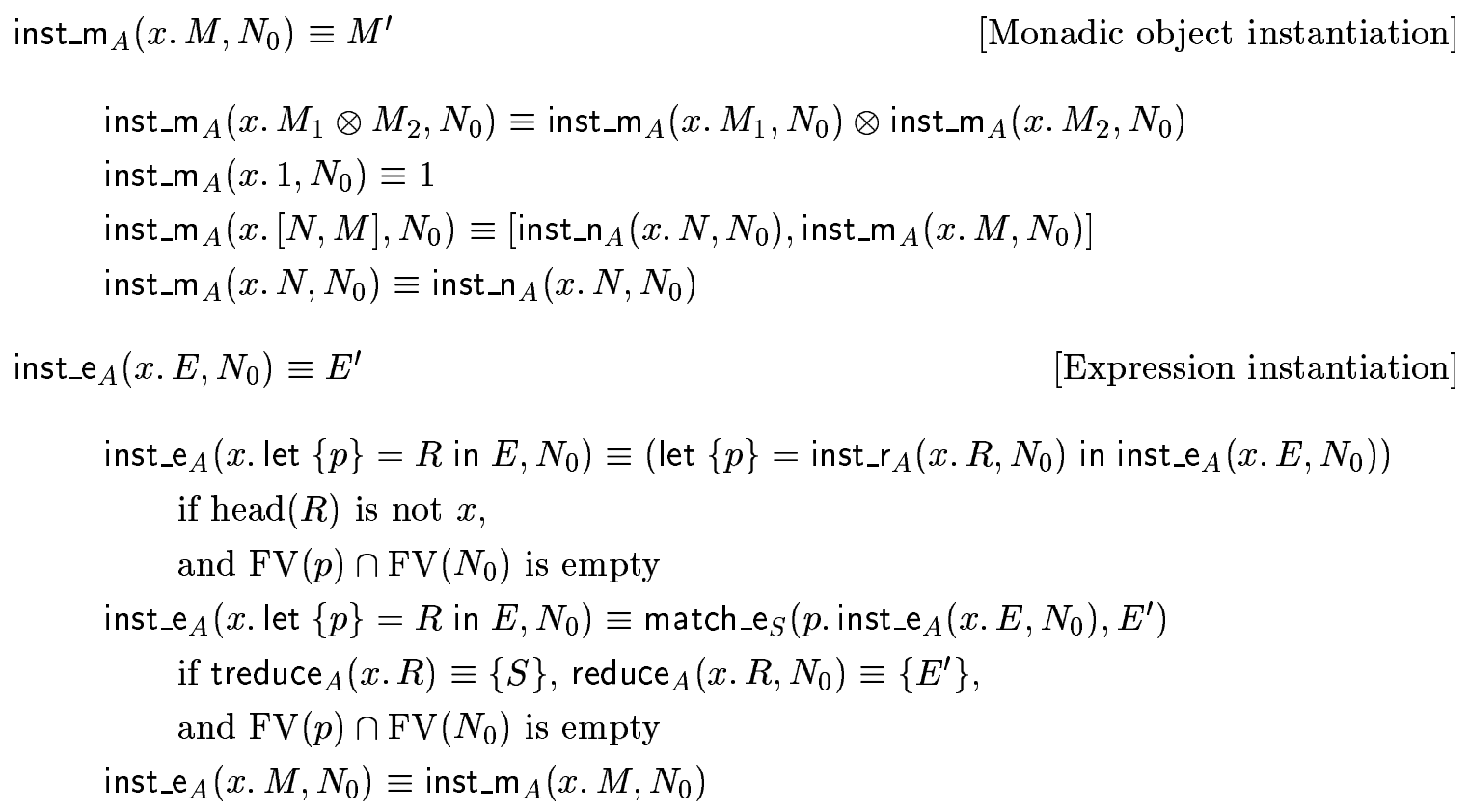

match_m $S_{1} \otimes S_{2}\left(p_{1} \otimes p_{2} . E, M_{1} \otimes M_{2}\right) \equiv$ match_m $_{S_{2}}\left(p_{2}\right.$. match_m $\left.S_{S_{1}}\left(p_{1} . E, M_{1}\right), M_{2}\right)$ if $\mathrm{FV}\left(p_{2}\right) \cap \mathrm{FV}\left(M_{1}\right)$ is empty

inst_p ${ }_{A}\left(x . P, N_{0}\right) \equiv P^{\prime}$

inst_a $_{A}\left(x . A, N_{0}\right) \equiv A^{\prime}$

inst_s $_{A}\left(x . S, N_{0}\right) \equiv S^{\prime}$

inst_k ${ }_{A}\left(x . K, N_{0}\right) \equiv K^{\prime}$
[Atomic type constructor instantiation]

[Type instantiation] [Synchronous type instantiation] [Kind instantiation]

\section{(Analogous.)}

\section{A.4 Expansion}

\section{DeFinition 23 (ExPANSION)}


$\operatorname{expand}_{A}(R) \equiv N$

[Expansion]

$\operatorname{expand}_{P}(R) \equiv R$

$\operatorname{expand}_{A \multimap B}(R) \equiv \hat{\lambda} x$. expand ${ }_{B}\left(R^{\wedge}\left(\operatorname{expand}_{A}(x)\right)\right) \quad$ if $x \notin \mathrm{FV}(R)$

expand $_{\Pi x: A . B}(R) \equiv \lambda x$. $\operatorname{expand}_{B}\left(R\left(\operatorname{expand}_{A}(x)\right)\right) \quad$ if $x \notin \mathrm{FV}(R)$

expand $_{A \& B}(R) \equiv\left\langle\operatorname{expand}_{A}\left(\pi_{1} R\right)\right.$, $\left.\operatorname{expand}_{B}\left(\pi_{2} R\right)\right\rangle$

$\operatorname{expand}_{\mathrm{T}}(R) \equiv\langle\rangle$

$\operatorname{expand}_{\{S\}}(R) \equiv\left(\right.$ let $\{p\}=R$ in $\left.\operatorname{pexpand}_{S}(p)\right)$

$\operatorname{pexpand}_{S}(p) \equiv M$

[Pattern expansion]

pexpand $_{S_{1} \otimes S_{2}}\left(p_{1} \otimes p_{2}\right) \equiv$ pexpand $_{S_{1}}\left(p_{1}\right) \otimes$ pexpand $_{S_{2}}\left(p_{2}\right)$

$\operatorname{pexpand}_{1}(1) \equiv 1$

$\operatorname{pexpand}_{\exists x: A . S}([x, p]) \equiv\left[\operatorname{expand}_{A}(x), \operatorname{pexpand}_{S}(p)\right]$

$\operatorname{pexpand}_{A}(x) \equiv \operatorname{expand}_{A}(x)$

\section{A.5 Typing}

Definition 24 (Signatures AND Contexts)

\begin{tabular}{|c|c|}
\hline$\Sigma::=\cdot|\Sigma, a: K| \Sigma, c: A$ & Signatures \\
\hline$\Gamma::=\cdot \mid \Gamma, x: A$ & Unrestricted contexts \\
\hline$\Delta::=\cdot \mid \Delta, x \hat{\wedge} A$ & Linear contexts \\
\hline$\Psi::=\cdot \mid p \bumpeq S, \Psi$ & Pattern contexts \\
\hline
\end{tabular}

DEFINITION 25 (TYPING)

$\vdash \Sigma$ ok

[Signature validity]

$$
\overline{\vdash \cdot \mathrm{ok}} \quad \frac{\vdash \Sigma \text { ok } \cdot \vdash_{\Sigma} K \Leftarrow \mathrm{kind}}{\vdash \Sigma, a: K \text { ok }} \quad \frac{\vdash \Sigma \text { ok } \cdot \vdash_{\Sigma} A \Leftarrow \text { type }}{\vdash \Sigma, c: A \text { ok }}
$$

$\vdash_{\Sigma} \Gamma$ ok

[Context validity]

$$
\overline{\vdash_{\Sigma} \cdot \mathrm{ok}} \quad \frac{\vdash_{\Sigma} \Gamma \text { ok } \Gamma \vdash_{\Sigma} A \Leftarrow \text { type }}{\vdash_{\Sigma} \Gamma, x: A \text { ok }}
$$

$\Gamma \vdash_{\Sigma} \Delta$ ok

[Linear context validity]

$$
\overline{\Gamma \vdash_{\Sigma} \cdot \text { ok }} \quad \frac{\Gamma \vdash_{\Sigma} \Delta \text { ok } \quad \Gamma \vdash_{\Sigma} A \Leftarrow \text { type }}{\Gamma \vdash_{\Sigma} \Delta, x \Uparrow A \text { ok }}
$$

$\Gamma \vdash_{\Sigma} \Psi$ ok

[Pattern context validity]

$\overline{\Gamma \vdash_{\Sigma} \cdot \mathrm{ok}}$

$\frac{\Gamma \vdash_{\Sigma} S \Leftarrow \text { type } \Gamma \vdash_{\Sigma} \Psi \text { ok }}{\Gamma \vdash_{\Sigma} p \wedge S, \Psi \text { ok }}$ 
$\Gamma \vdash_{\Sigma} K \Leftarrow$ kind

[Kind checking]

$$
\overline{\Gamma \vdash \text { type } \Leftarrow \text { kind }} \text { typeKF } \quad \frac{\Gamma \vdash A \Leftarrow \text { type } \Gamma, x: A \vdash K \Leftarrow \text { kind }}{\Gamma \vdash \Pi x: A . K \Leftarrow \text { kind }} \Pi \mathbf{K F}
$$

$\Gamma \vdash_{\Sigma} A \Leftarrow$ type

[Type checking]

$$
\begin{gathered}
\frac{\Gamma \vdash A \Leftarrow \text { type } \quad \Gamma, x: A \vdash B \Leftarrow \text { type }}{\Gamma \vdash \Pi x: A . B \Leftarrow \text { type }} \Pi \quad \frac{\Gamma \vdash P \Rightarrow \text { type }}{\Gamma \vdash P \Leftarrow \text { type }} \Rightarrow \text { type } \Leftarrow \\
\frac{\Gamma \vdash A \Leftarrow \text { type } \quad \Gamma \vdash B \Leftarrow \text { type }}{\Gamma \vdash A \multimap B \Leftarrow \text { type }} \multimap \mathbf{F} \\
\frac{\Gamma \vdash A \Leftarrow \text { type } \Gamma \vdash B \Leftarrow \text { type }}{\Gamma \vdash A \& B} \quad \overline{\mathbf{F}} \quad \overline{\Gamma \vdash \top \Leftarrow \text { type }} \top \mathbf{F} \\
\frac{\Gamma \vdash S \Leftarrow \text { type }}{\Gamma \vdash\{S\} \Leftarrow \text { type }}\{\} \mathbf{F}
\end{gathered}
$$

$\Gamma \vdash_{\Sigma} S \Leftarrow$ type

[Synchronous type checking]

$$
\begin{gathered}
\frac{\Gamma \vdash S_{1} \Leftarrow \text { type } \quad \Gamma \vdash S_{2} \Leftarrow \text { type }}{\Gamma \vdash S_{1} \otimes S_{2} \Leftarrow \text { type }} \otimes \mathbf{F} \quad \overline{\Gamma \vdash 1 \Leftarrow \text { type }} 1 \mathbf{F} \\
\frac{\Gamma \vdash A \Leftarrow \text { type } \quad \Gamma, x: A \vdash S \Leftarrow \text { type }}{\Gamma \vdash \exists x: A . S \Leftarrow \text { type }} \exists \mathbf{F}
\end{gathered}
$$

$\Gamma \vdash_{\Sigma} P \Rightarrow K$

[Atomic type constructor inference]

$$
\frac{\Gamma \vdash P \Rightarrow \Pi x: A . K \quad \Gamma ; \cdot \vdash N \Leftarrow A}{\Gamma \vdash a \Rightarrow \Sigma(a)} a \quad \Pi \mathbf{K E}
$$

$\Gamma \vdash_{\Sigma} N \Leftarrow A$

[Normal object checking]

$$
\begin{aligned}
& \frac{\Gamma, x: A ; \Delta \vdash N \Leftarrow B}{\Gamma ; \Delta \vdash \lambda x . N \Leftarrow \Pi x: A . B} \Pi \mathbf{\Pi} \quad \frac{\Gamma ; \Delta \vdash R \Rightarrow P^{\prime} \quad P^{\prime}=P}{\Gamma ; \Delta \vdash R \Leftarrow P} \Rightarrow \Leftarrow \\
& \frac{\Gamma ; \Delta, x \triangleq A \vdash N \Leftarrow B}{\Gamma ; \Delta \vdash \hat{\lambda} x . N \Leftarrow A \multimap B} \multimap \mathbf{I} \\
& \frac{\Gamma ; \Delta \vdash N_{1} \Leftarrow A \quad \Gamma ; \Delta \vdash N_{2} \Leftarrow B}{\Gamma ; \Delta \vdash\left\langle N_{1}, N_{2}\right\rangle \Leftarrow A \& B} \& \mathbf{I} \quad \overline{\Gamma ; \Delta \vdash\langle\rangle \Leftarrow \top} \top \mathbf{I} \\
& \frac{\Gamma ; \Delta \vdash E \leftarrow S}{\Gamma ; \Delta \vdash\{E\} \Leftarrow\{S\}}\{\} \mathbf{I}
\end{aligned}
$$

$\Gamma \vdash_{\Sigma} R \Rightarrow A$

[Atomic object inference]

$$
\begin{aligned}
& \overline{\Gamma ; \cdot \vdash c \Rightarrow \Sigma(c)}^{c} \quad \overline{\Gamma ; \cdot \vdash x \Rightarrow \Gamma(x)}^{x} \quad \frac{\Gamma ; \Delta \vdash R \Rightarrow \Pi x: A . B \quad \Gamma ; \cdot \vdash N \Leftarrow A}{\Gamma ; \Delta \vdash R N \Rightarrow \text { inst_a }_{A}(x . B, N)} \Pi \mathbf{E} \\
& \overline{\Gamma ; x} \wedge A \vdash x \Rightarrow A^{\wedge} \quad \frac{\Gamma ; \Delta_{1} \vdash R \Rightarrow A \multimap B \quad \Gamma ; \Delta_{2} \vdash N \Leftarrow A}{\Gamma ; \Delta_{1}, \Delta_{2} \vdash R^{\wedge} N \Rightarrow B} \multimap \mathbf{E} \\
& \frac{\Gamma ; \Delta \vdash R \Rightarrow A \& B}{\Gamma ; \Delta \vdash \pi_{1} R \Rightarrow A} \& \mathbf{E}_{1} \quad \frac{\Gamma ; \Delta \vdash R \Rightarrow A \& B}{\Gamma ; \Delta \vdash \pi_{2} R \Rightarrow B} \& \mathbf{E}_{2}
\end{aligned}
$$


$\Gamma ; \Delta \vdash_{\Sigma} E \leftarrow S$

[Expression checking]

$$
\frac{\Gamma ; \Delta_{1} \vdash R \Rightarrow\left\{S_{0}\right\} \quad \Gamma ; \Delta_{2} ; p \bumpeq S_{0} \vdash E \leftarrow S}{\Gamma ; \Delta_{1}, \Delta_{2} \vdash(\text { let }\{p\}=R \text { in } E) \leftarrow S}\{\} \mathbf{E} \quad \frac{\Gamma ; \Delta \vdash M \Leftarrow S}{\Gamma ; \Delta \vdash M \leftarrow S} \Leftarrow \leftarrow
$$

$\Gamma ; \Delta ; \Psi \vdash_{\Sigma} E \leftarrow S$

[Pattern expansion]

$$
\begin{aligned}
& \frac{\Gamma ; \Delta \vdash E \leftarrow S}{\Gamma ; \Delta ; \cdot \vdash E \leftarrow S} \leftarrow \leftarrow
\end{aligned}
$$

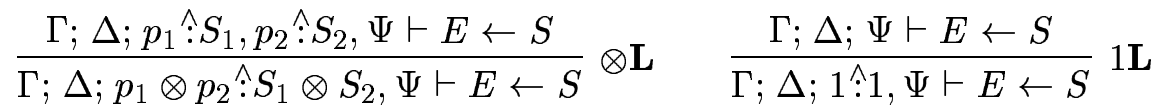

$$
\begin{aligned}
& \frac{\Gamma, x: A ; \Delta ; p \wedge S_{0}, \Psi \vdash E \leftarrow S}{\Gamma ; \Delta ;[x, p]: \exists x: A . S_{0}, \Psi \vdash E \leftarrow S} \exists \mathbf{L} \quad \frac{\Gamma ; \Delta, x \wedge A ; \Psi \vdash E \leftarrow S}{\Gamma ; \Delta ; x \wedge A, \Psi \vdash E \leftarrow S} \mathrm{AL}
\end{aligned}
$$

$\Gamma ; \Delta \vdash_{\Sigma} M \Leftarrow S$

[Monadic object checking]

$$
\begin{gathered}
\frac{\Gamma ; \Delta_{1} \vdash M_{1} \Leftarrow S_{1} \quad \Gamma ; \Delta_{2} \vdash M_{2} \Leftarrow S_{2}}{\Gamma ; \Delta_{1}, \Delta_{2} \vdash M_{1} \otimes M_{2} \Leftarrow S_{1} \otimes S_{2}} \otimes \mathbf{I} \quad \overline{\Gamma ; \cdot \vdash 1 \Leftarrow 1} 1 \mathbf{I} \\
\frac{\Gamma ; \cdot \vdash N \Leftarrow A \quad \Gamma ; \Delta \vdash M \Leftarrow \text { inst_s } A(x . S, N)}{\Gamma ; \Delta \vdash[N, M] \Leftarrow \exists x: A . S} \exists \mathbf{I}
\end{gathered}
$$

\section{References}

[Abr93] Samson Abramsky. Computational interpretations of linear logic. Theoretical Computer Science, 111(1-2):3-57, 1993.

[And92] Jean-Marc Andreoli. Logic programming with focusing proofs in linear logic. Journal of Logic and Computation, 2(3):197-347, 1992.

[AP91] Jean-Marc Andreoli and Remo Pareschi. Linear objects: Logical processes with built-in inheritance. New Generation Computing, 9:445-473, 1991.

[Bar96] Andrew Barber. Dual intuitionistic linear logic. Technical Report ECS-LFCS96-347, Department of Computer Science, University of Edinburgh, September 1996.

[Bar97] Andrew Graham Barber. Linear Type Theories, Semantics and Action Calculi. PhD thesis, University of Edinburgh, 1997. Available as Technical Report ECSLFCS-97-371.

[BM01] David Basin and Seán Matthews. Logical frameworks. In Dov Gabbay and Franz Guenthner, editors, Handbook of Philosophical Logic. Kluwer Academic Publishers, 2nd edition, 2001. In preparation.

[BS94] G. Bellin and P. J. Scott. On the $\pi$-calculus and linear logic. Theoretical Computer Science, 135:11-65, 1994.

[Cer96] Iliano Cervesato. A Linear Logical Framework. PhD thesis, Dipartimento di Informatica, Università di Torino, February 1996. 
[Chi95] Jawahar Lal Chirimar. Proof Theoretic Approach to Specification Languages. $\mathrm{PhD}$ thesis, University of Pennsylvania, May 1995.

[Coq91] Thierry Coquand. An algorithm for testing conversion in type theory. In Gérard Huet and Gordon Plotkin, editors, Logical Frameworks, pages 255-279. Cambridge University Press, 1991.

[CP98] Iliano Cervesato and Frank Pfenning. A linear logical framework. Information and Computation, 1998. To appear in a special issue with invited papers from LICS'96, E. Clarke, editor.

[CPWW02] Iliano Cervesato, Frank Pfenning, David Walker, and Kevin Watkins. A concurrent logical framework II: Examples and applications. Technical Report CMU-CS-02-102, Department of Computer Science, Carnegie Mellon University, 2002. Forthcoming.

[dB80] N.G. de Bruijn. A survey of the project AUTOMATH. In J.P. Seldin and J.R. Hindley, editors, To H.B. Curry: Essays in Combinatory Logic, Lambda Calculus and Formalism, pages 579-606. Academic Press, 1980.

[Fel91] Amy Felty. Encoding dependent types in an intuitionistic logic. In Gérard Huet and Gordon D. Plotkin, editors, Logical Frameworks, pages 214-251. Cambridge University Press, 1991.

[FM97] M. Fairtlough and M.V. Mendler. Propositional lax logic. Information and Computation, 137(1):1-33, August 1997.

[Gha97] Neil Ghani. Eta-expansions in dependent type theory - the calculus of constructions. In P. de Groote and J.R. Hindley, editors, Proceedings of the Third International Conference on Typed Lambda Calculus and Applications (TLCA'97), pages 164-180, Nancy, France, April 1997. Springer-Verlag LNCS 1210 .

[Gir87] J.-Y. Girard. Linear logic. Theoretical Computer Science, 50:1-102, 1987.

[Gog94] Healfdene Goguen. A Typed Operational Semantics for Type Theory. PhD thesis, Edinburgh University, August 1994.

[Gog99] Healfdene Goguen. Soundness of the logical framework for its typed operational semantics. In Jean-Yves Girard, editor, Proceedings of the 4 th International Conference on Typed Lambda Calculi and Applications (TLCA'99), pages 177197, L'Aquila, Italy, April 1999. Springer-Verlag LNCS 1581.

[HHP93] Robert Harper, Furio Honsell, and Gordon Plotkin. A framework for defining logics. Journal of the Association for Computing Machinery, 40(1):143-184, January 1993.

[HM94] Joshua Hodas and Dale Miller. Logic programming in a fragment of intuitionistic linear logic. Information and Computation, 110(2):327-365, 1994. A preliminary version appeared in the Proceedings of the Sixth Annual IEEE Symposium on Logic in Computer Science, pages 32-42, Amsterdam, The Netherlands, July 1991. 
[HMS01] Furio Honsell, Marino Miculan, and Ivan Scagnetto. Pi-calculus in (co)inductive type theories. Theoretical Computer Science, 253(2):239-285, 2001.

[HP00] Robert Harper and Frank Pfenning. On equivalence and canonical forms in the LF type theory. Technical Report CMU-CS-00-148, Department of Computer Science, Carnegie Mellon University, July 2000.

[HPW96] James Harland, David Pym, and Michael Winikoff. Programming in Lygon: An overview. In M. Wirsing and M. Nivat, editors, Proceedings of the Fifth International Conference on Algebraic Methodology and Software Technology, pages 391-405, Munich, July 1996. Springer-Verlag LNCS 1101.

[HWTK98] Joshua S. Hodas, Kevin M. Watkins, Naoyuki Tamura, and Kyoung-Sun Kang. Efficient implementation of a linear logic programming language. In Proceedings of the Joint International Conference and Symposium on Logic Programming, pages 145-159, Manchester, June 1998.

[IP98] Samin Ishtiaq and David Pym. A relevant analysis of natural deduction. Journal of Logic and Computation, 8(6):809-838, 1998.

[KY93] Naoki Kobayashi and Akinori Yonezawa. ACL — A concurrent linear logic programming paradigm. In D. Miller, editor, Proceedings of the 1993 International Logic Programming Symposium, pages 279-294, Vancouver, Canada, 1993. MIT Press.

[Mil92] Dale Miller. The $\pi$-calculus as a theory in linear logic: Preliminary results. In E. Lamma and P. Mello, editors, Proceedings of the Workshop on Extensions of Logic Programming, pages 242-265. Springer-Verlag LNCS 660, 1992.

[Mil96] Dale Miller. Forum: A multiple-conclusion specification logic. Theoretical Computer Science, 165(1):201-232, 1996.

[ML96] Per Martin-Löf. On the meanings of the logical constants and the justifications of the logical laws. Nordic Journal of Philosophical Logic, 1(1):11-60, 1996.

[MNPS91] Dale Miller, Gopalan Nadathur, Frank Pfenning, and Andre Scedrov. Uniform proofs as a foundation for logic programming. Annals of Pure and Applied Logic, 51:125-157, 1991.

[Mog89] Eugenio Moggi. Computational lambda calculus and monads. In Proceedings of the Fourth Symposium on Logic in Computer Science, pages 14-23, Asilomar, California, June 1989. IEEE Computer Society Press.

[Mog91] Eugenio Moggi. Notions of computation and monads. Information and Computation, 93(1):55-92, 1991.

[MOM91] N. Martí-Oliet and J. Meseguer. From Petri nets to linear logic through categories: A survey. Journal on Foundations of Computer Science, 2(4):297-399, December 1991. 
[PD01] Frank Pfenning and Rowan Davies. A judgmental reconstruction of modal logic. Mathematical Structures in Computer Science, 11(4):511-540, August 2001.

[PE88] Frank Pfenning and Conal Elliott. Higher-order abstract syntax. In Proceedings of the ACM SIGPLAN '88 Symposium on Language Design and Implementation, pages 199-208, Atlanta, Georgia, June 1988.

[Per98] Guy Perrier. Concurrent programming as proof net construction. Mathematical Structures in Computer Science, 8(6):681-710, 1998.

[Pet62] Carl Adam Petri. Kommunikation mit Automaten. Bonn: Institut für Instrumentelle Mathematik, Schriften des IIM Nr. 2, 1962.

[Pfe91] Frank Pfenning. Logic programming in the LF logical framework. In Gérard Huet and Gordon Plotkin, editors, Logical Frameworks, pages 149-181. Cambridge University Press, 1991.

[Pfe99] Frank Pfenning. Logical frameworks. In Alan Robinson and Andrei Voronkov, editors, Handbook of Automated Reasoning. Elsevier Science Publishers, 1999. In preparation.

[Pfe00] Frank Pfenning. Structural cut elimination: I. Intuitionistic and classical logic. Information and Computation, 157(1/2):84-141, March 2000.

[Pfe01a] Frank Pfenning. Intensionality, extensionality, and proof irrelevance in modal type theory. In J. Halpern, editor, Proceedings of the 16th Annual Symposium on Logic in Computer Science (LICS'01), pages 221-230, Boston, MA, June 2001. IEEE Computer Society Press.

[Pfe01b] Frank Pfenning. Logical frameworks. In Alan Robinson and Andrei Voronkov, editors, Handbook of Automated Reasoning, chapter 16, pages 977-1061. Elsevier Science and MIT Press, 2001. In press.

[Pra65] Dag Prawitz. Natural Deduction. Almquist \& Wiksell, Stockholm, 1965.

[VC00] Joseph C. Vanderwaart and Karl Crary. A simplified account of the metatheory of linear LF. Draft paper, September 2000. 